Japan. J. Math.

Vol. 31, No. 1, 2005

\title{
A new complexity for 3-manifolds
}

\author{
By Mariko ENDOH and Ippei IsHII \\ (Received November 14, 2003) \\ (Revised March 16, 2004) \\ (from Tokyo Journal of Mathematics)
}

\section{Introduction}

There are some well known numbers which estimate how complex a 3-manifold $M$ is, for example, the algebraic rank $r k(M)$ which gives the least number of generators of the fundamental group $\pi_{1}(M)$, and the Heegaard genus $H G(M)$ which gives the smallest genus among all the Heegaard splittings of $M$. In this paper, we will propose a new complexity, called a block number, for oriented closed 3-manifolds. A pair $\mathfrak{C}=(M, X)$ of an orientable closed 3-manifold $M$ and a non-singular vector field $X$ on it is called an oriented combed manifold. A block number is defined not only for a 3-manifold but also for an oriented combed 3-manifold.

A block number of an orientable closed 3-manifold $M$ is denoted by $B l(M)$, and a block number of an oriented combed 3-manifold $\mathfrak{C}$ defined on $M$ is denoted by $B l^{*}(\mathfrak{C})$. Both $B l(M)$ and $B l^{*}(\mathfrak{C})$ are defined by means of flow-spines (cf. [8]) or DS-diagrams with E-cycle (cf. [6]). The block numbers $B l(M)$ and $B l^{*}(\mathfrak{C})$ are so defined that

$$
B l(M)=\min \left\{B l^{*}(\mathfrak{C}) \mid \mathfrak{C} \text { is an oriented combed 3-manifold on } M\right\} .
$$

It will be shown in $\S 3$ that an oriented combed 3-manifold $\mathfrak{C}=(L(p, q), X)$ on a lens space $L(p, q)$ satisfies $B l^{*}(\mathfrak{C})=B l(L(p, q))$ if and only if the non-singular vector field $X$ is homotopic to the vector field everywhere tangential to a fiber of a Seifert fibration of $L(p, q)$. This fact shows that the block number $B l^{*}(\mathfrak{C})$ really refines the block number $B l(M)$.

We will show in $\S 2$ some basic properties of block numbers, for example, $B l\left(S^{2} \times S^{1}\right)=0, B l\left(S^{3}\right)=1$ and $H G(M) \leq B l(M)$ for any $M \neq S^{2} \times S^{1}$. In $\S 4$, we will consider the Seifert fibered space on $S^{2}$ with three exceptional fibers, and show that $B l^{*}(\mathfrak{C})=2$ for a canonical oriented combed 3-manifold on such a Seifert fibered space.

There remain many interesting problems concerning with block numbers. We 
will exhibit in $\S 5$ some remaining problems.

\section{Preliminaries and definitions}

The notion of fake surfaces, DS-diagrams, and DS-diagrams with E-cycle were introduced by H. Ikeda ([2]-[6]). In this section, we shall review these concepts, and define the block number for an orientable closed 3-manifold and for an oriented combed 3-manifold (cf. Definition 1.2 below).

1.1. Fake surfaces, simple spines and DS-diagrams. A finite 2dimensional polyhedron $P$ is called a closed fake surface, if any point has a neighborhood homeomorphic to an open subset of

$$
\left(\mathbb{R}^{2} \times\{0\}\right) \cup\left(\mathbb{R} \times\{0\} \times \mathbb{R}_{+}\right) \cup\left(\{0\} \times \mathbb{R} \times \mathbb{R}_{-}\right) \subset \mathbb{R}^{3},
$$

that is, the shape of a neighborhood of a point $x \in P$ is one of the three types shown in Figure 1.1.
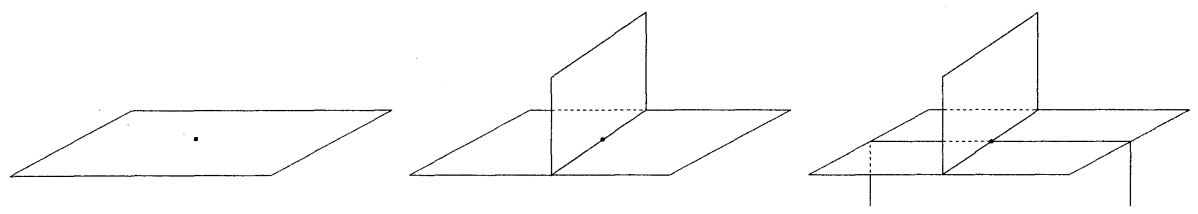

Figure 1.1. Neighborhood of a point on a fake surface

A closed fake surface $P$ is naturally stratified as $V(P) \subset S(P) \subset P$, where $V(P)$ is a finite set of points, called vertices, which play the role of 0 in the above subset of $\mathbb{R}^{3}$, and $S(P)$ is the singular set which consists of the points playing the role of $(t, 0,0)$ or $(0, t, 0)$. A connected component of $P-S(P)$ is called a face of $P$, and a connected component of $S(P)-V(P)$ is called an edge of $P$.

REMARK 1.1. We do not assume that the stratification of a closed fake surface is cellular, namely, a face may not be an open 2-disk, and an edge may not be an open arc but may be a circle.

If a closed fake surface $P$ is embedded in a closed 3-manifold $M$ so that $M-P$ is homeomorphic to an open 3-ball, then we call $P$ a simple spine of $M$. Cutting out a closed 3-manifold $M$ along its simple spine $P$, we obtain a 3 -ball $B^{3}$ with a gluing map on its boundary $S^{2}=\partial B^{3}$. We can formulate this situation as the following definition of a DS-diagram.

Definition 1.1. A triple $\Delta=(G, f, P)$ is called a $D S$-diagram if

(1) $G$ is a 3 -regular graph embedded in the 2-sphere $S^{2}$, 
(2) $P$ is a closed fake surface,

(3) $f$ is a local homeomorphism from $S^{2}$ onto $P$ such that $f^{-1}(S(P))=G$ and $f^{-1}(V(P))=V_{G}$, where $V_{G}$ is the set of the vertices of the graph $G$, and

(4) $\# f^{-1}(x)=4$ for any $x \in V(P), \# f^{-1}(x)=3$ for any $x \in S(P)-V(P)$, and $\# f^{-1}(x)=2$ for any $x \in P-S(P)$.

REMARK 1.2. The graph $G$ of a DS-diagram in our situation may contain a hoop, a loop without any vertex on it (cf. REMARK 1.1). The diagram shown in Figure 1.2, which represents $S^{2} \times S^{1}$, is an example of such a DS-diagram. Among DS-diagrams with E-cycle defined below, this is the only example containing a hoop.

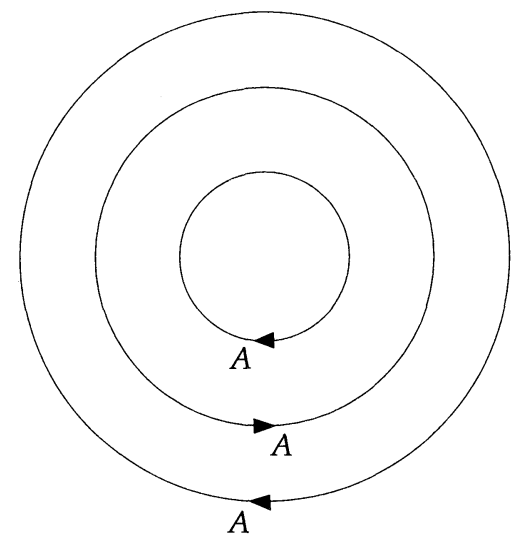

Figure 1.2. DS-diagram with a hoop

Let $B^{3}$ be a 3 -ball with boundary $\partial B^{3}=S^{2}$ on which a DS-diagram $\Delta=$ $(G, f, P)$ is given. Then, gluing $\partial B^{3}$ by the map $f$, we get a closed 3 -manifold $M(\Delta)=B^{3} / f$ with a simple spine $P=f\left(S^{2}\right)$.

1.2. Oriented combed 3-manifolds. In this paper, we deal with an oriented closed 3-manifold equipped with a non-singular vector field on it. We denote by $\mathfrak{X}_{0}(M)$ the set of all non-singular vector fields on a closed 3-manifold $M$, and, for a non-singular vector field $X \in \mathfrak{X}_{0}(M),[X]$ denotes the homotopy class of $X$ within $\mathfrak{X}_{0}(M)$. Oriented combed 3-manifolds and their equivalence are defined as follows.

Definition 1.2. An oriented combed 3-manifold $\mathfrak{C}=(M, X)$ is a pair of an oriented closed 3-manifold $M$ and a non-singular vector field $X$ on $M$. Two oriented combed 3 -manifolds $\mathfrak{C}_{j}=\left(M_{j}, X_{j}\right)(j=1,2)$ are said to be mutually equivalent, written by $\mathfrak{C}_{1} \cong \mathfrak{C}_{2}$, if there is an orientation preserving diffeomorphism 
$h: M_{1} \rightarrow M_{2}$ such that $\left[h_{*}\left(X_{1}\right)\right]=\left[X_{2}\right]$.

In what follows, we always assume that the closed 3-manifold $M=M(\Delta)$ represented by a DS-diagram $\Delta$ is orientable.

1.3. DS-diagrams with E-cycle. A cycle $c=\left\{E_{1}, E_{2}, \ldots, E_{\nu}\right\}$ of a graph $G$ is a sequence of edges $E_{j}\left(E_{i} \neq E_{j}\right.$ if $\left.i \neq j\right)$ such that the closure of $\bigcup_{j} E_{j}$ is a simple closed curve.

Definition 1.3. Let $\Delta=(G, f, P)$ be a DS-diagram, $e$ be a cycle of $G$ (the underlying space of the closure of the cycle $e$ is denoted by the same letter $e$ ), and $\Sigma_{k}(k=1,2)$ be the connected components of $S^{2}-e$. The cycle $e$ of the graph $G$ is said to be an $E$-cycle, if it satisfies that

(1) $\#\left(e \cap f^{-1}(x)\right)=2$ for any $x \in V(P)$,

(2) $\#\left(e \cap f^{-1}(x)\right)=1$ for any $x \in S(P)-V(P)$, and

(3) the restriction of $f$ onto $\Sigma_{k}$ is a bijection for each $k=1,2$.

There exists a DS-diagram which admits some different E-cycles. When we consider a DS-diagram with E-cycle, we always assume that an E-cycle $e$ is specified, and has a fixed orientation (an E-cycle given the reversed orientation is denoted by $\bar{e}$ ). Moreover we assume that $S^{2}$ on which the graph $G$ is drawn is oriented, and so each component $\Sigma_{k}(k=1,2)$ of $S^{2}-e$ has the restricted orientation. The component $\Sigma_{k}$ with $e=\partial \Sigma_{k}$ (in the oriented sense) is called the positive region. We represent a DS-diagram with E-cycle by a four-tuple $\Delta=(G, f, P ; e)$. A DSdiagram having the reversed orientation on $S^{2}$ and the same orientation on $e$ is called the conjugate of $\Delta$, and is denoted by $\Delta^{*}$. It is known that any closed 3 manifold can be represented by a DS-diagram with E-cycle ([6], [8]), and moreover it is known that, in the orientable case, a DS-diagram with E-cycle defines not only a closed manifold but also an oriented combed manifold (see [8] and [11]).

Let $\Delta=(G, f, P ; e)$ be a DS-diagram with an E-cycle $e$. We may assume that

$$
\begin{aligned}
& B^{3}=\left\{(x, y, z) \in \mathbb{R}^{3} \mid x^{2}+y^{2}+z^{2} \leq 1\right\}, \quad G \subset S^{2} \equiv \partial B^{3}, \\
& e=S^{2} \cap\{z=0\} \quad \text { (the equator). }
\end{aligned}
$$

The E-cycle $e$ divides $S^{2}$ into two open 2-disks $\Sigma^{+}$and $\Sigma^{-}$, that is,

$$
\Sigma^{+}=S^{2} \cap\{z>0\} \quad \text { and } \quad \Sigma^{-}=S^{2} \cap\{z<0\} .
$$

The 3-ball $B^{3}$ is always assumed to be given the usual orientation so that it is compatible with the orientation of $S^{2}=\partial B^{3}$, on which an orientation was already fixed by a DS-diagram with E-cycle. Moreover we assume that the orientation on the E-cycle $e$ is compatible with the orientation on $S^{2}$ restricted to $\Sigma^{+}$, that is, $\Sigma^{+}$is the positive region. Then we get an oriented manifold $M(\Delta)=B^{3} / f$ and a 
non-singular vector field $X(\Delta)=(\partial / \partial z) / f$ on $M(\Delta)$ (cf. [9]). Hence $\Delta$ determines an oriented combed 3-manifold $\mathfrak{C}(\Delta)=(M(\Delta), X(\Delta))$.

REMARK 1.3. The oriented combed manifold $\mathfrak{C}\left(\Delta^{*}\right)$ represented by the conjugate $\Delta^{*}$ is equivalent to $(M(\Delta),-X(\Delta))$, the same oriented 3-manifold equipped with the reversed vector field. Moreover $\bar{\Delta}=(G, f, P ; \bar{e})$, which is different from $\Delta$ only by the orientation on the E-cycle, represents the reversed vector field on the orientation reversed manifold $-M(\Delta)$, namely the oriented combed 3-manifold $(M(\bar{\Delta}), X(\bar{\Delta}))$ is equivalent to $(-M(\Delta),-X(\Delta))$.

1.4. E-data and block numbers. Let $\Delta=(G, f, P ; e)$ be a DS-diagram with E-cycle, which is assumed to be given on the unit sphere in $\boldsymbol{R}^{3}$ as in the previous subsection. For each vertex $v \in V(P)$ of the spine $P=\partial B^{3} / f$, there are exactly two vertices $v^{+}$and $v^{-}$of the graph $G$ such that $f\left(v^{+}\right)=f\left(v^{-}\right)=v$, and $v^{+}$and $v^{-}$are both on the E-cycle $e$. These two vertices are characterized by the condition

(*) $U \cap(G-e) \subset \Sigma^{+}$(or $\Sigma^{-}$) for sufficiently small neighborhood $U$ (in $S^{2}$ ) of $v^{+}$ (respectively $v^{-}$).

Each vertex $v \in V(P)$ is classified into the two cases $(\ell)$ or $(r)$ shown in Figure 1.3 (cf. [8] and [11]). We define the code $\phi(v)$ so that $\phi(v)=\ell$ (or $r$ ) if $v$ is the vertex of type $(\ell)$ (respectively $(r)$ ).

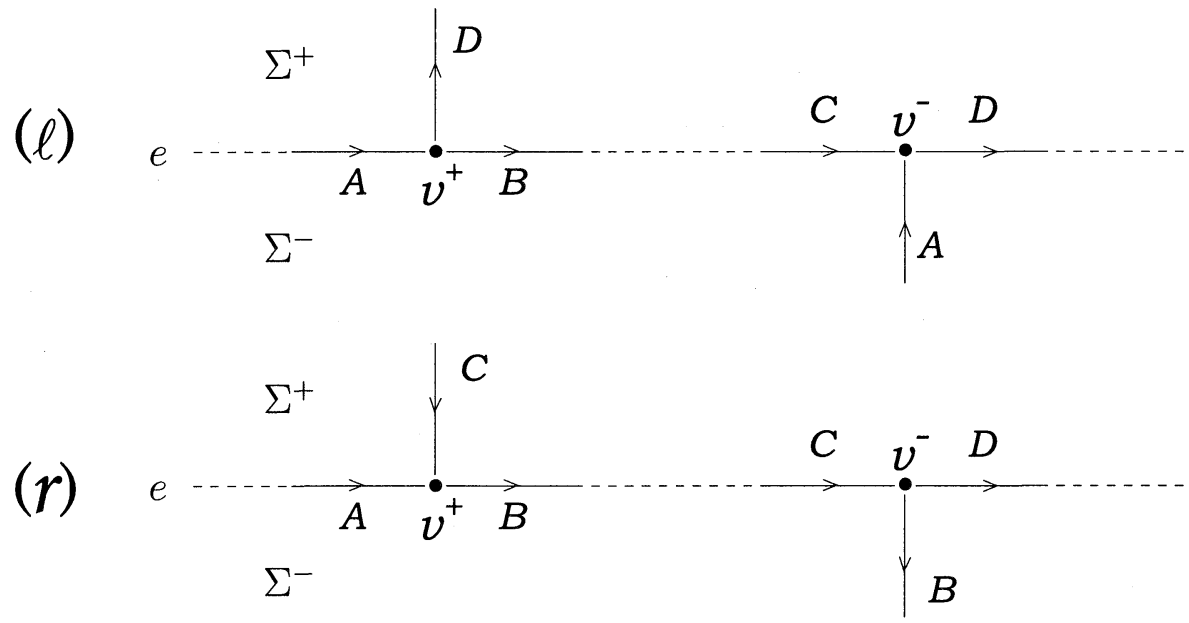

Figure 1.3. The code $\phi(v)=\ell$ or $r$ 
Suppose that $V(P)$ consists of $n$ points $v_{1}, v_{2}, \ldots, v_{n}$. An $E$-data

$$
\mathcal{E}(\Delta)=(\phi, \mathcal{A}(\Delta))
$$

of $\Delta=(G, f, P ; e)$ is a pair consisting of the code $\phi\left(v_{k}\right)$ for each $v_{k} \in V(P)$ and the arrangement $\mathcal{A}(\Delta)$ of $2 n$ points $v_{k}^{ \pm}$on the oriented circle $S^{1} \equiv e$.

REMARK 1.4. It is to be noticed that not all E-data correspond to a DSdiagram. An E-data which does not represent any DS-diagram is shown in [8] (such an E-data is called a "singularity-data" in [8]). An E-data is said to be realizable if it is really an E-data of a DS-diagram with E-cycle. However it is shown in [7] that any E-data, even if it is not realizable, represents a compact manifold with boundary, and an E-data corresponds to a DS-diagram if the boundary of the represented compact manifold is a 2 -sphere.

It is known that an E-data, if it is realizable, completely determines a DSdiagram with E-cycle, and there are several methods for representing an E-data (see [8], [11], [12], and see also [1]). In this paper, we use a new convenient representation, called a coded sequence, which is explained in the following example.

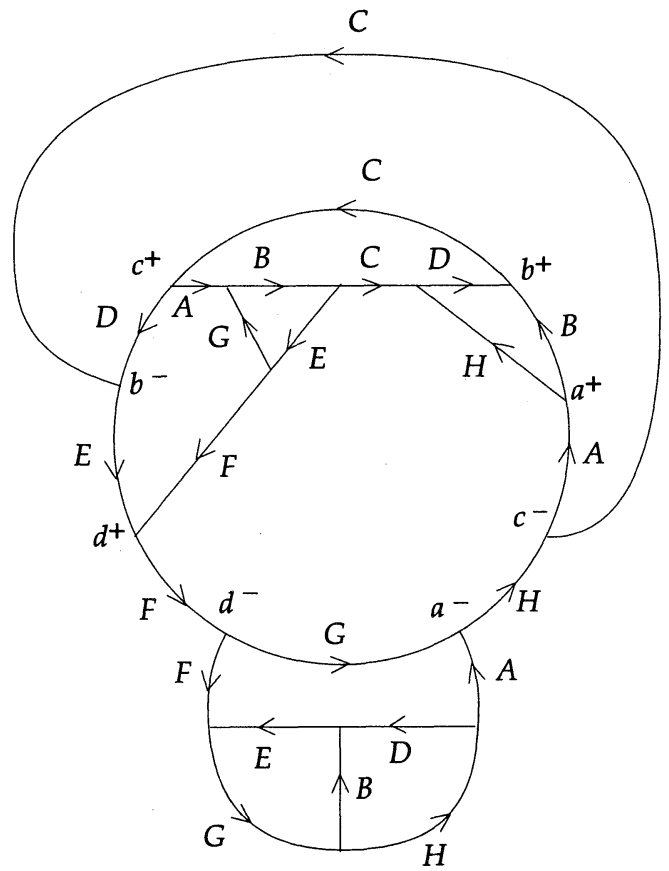

Figure 1.4 
ExAmple 1.1. Let $\Delta=(G, f, P ; e)$ be the DS-diagram drawn in Figure 1.4, which has four vertices $V(P)=\{a, b, c, d\}$. The codes of these vertices are $\phi(a)=$ $\phi(c)=\ell$ and $\phi(b)=\phi(d)=r$, and the arrangement $\mathcal{A}(\Delta)$ is given by a sequence

$$
\mathcal{A}(\Delta)=\left\{a^{+} b^{+} c^{+} b^{-} d^{+} d^{-} a^{-} c^{-}\right\}
$$

which indicates the cyclical order of the points in $V(G) \cap e$. Of course we may employ a cyclically permuted sequence for representing the arrangement. The Edata $\mathcal{E}(\Delta)$ is represented by the following coded sequence:

$$
\mathcal{E}(\Delta)=\left\{a^{+\ell} b^{+r} c^{+\ell} b^{-r} d^{+r} d^{-r} a^{-\ell} c^{-\ell}\right\}
$$

We call a consecutive (in the sense of cyclical order) sequence of symbols in $\mathcal{A}(\Delta)$ a subword of an arrangement $\mathcal{A}(\Delta)$. If any symbol in a subword $W$ has the signature $+($ or - ), then we call $W$ a positive subword (respectively a negative subword).

Definition 1.4. A maximal positive (or negative) subword of an arrangement $\mathcal{A}(\Delta)$ is called a positive block (respectively a negative block) of $\mathcal{A}(\Delta)$.

For example, the arrangement $\mathcal{A}(\Delta)$ in Example 1.1 has two positive blocks $W_{1}^{+}=a^{+} b^{+} c^{+}, W_{2}^{+}=d^{+}$and two negative blocks $W_{3}^{-}=b^{-}, W_{4}^{-}=d^{-} a^{-} c^{-}$, and we can represent $\mathcal{A}(\Delta)$ as $\mathcal{A}(\Delta)=W_{1}^{+} W_{3}^{-} W_{2}^{+} W_{4}^{-}$. Also for a coded sequence we can define the notion of a "subword", a "positive subword" and a "negative subword".

The block number of a DS-diagram with E-cycle, one of an orientable closed 3-manifold, and one of an oriented combed 3-manifolds are defined as follow.

Definition 1.5. The block number $b l(\Delta)$ of a DS-diagram $\Delta$ with E-cycle is defined to be the number of positive blocks included in the arrangement $\mathcal{A}(\Delta)$.

Definition 1.6. The block number $B l(M)$ of a orientable closed 3-manifold $M$ is defined by

$$
\begin{aligned}
B l(M)=\min \{b l(\Delta) \mid \Delta & \text { is a DS-diagram with E-cycle } \\
& \text { such that } M(\Delta) \text { is homeomorphic to } M\} .
\end{aligned}
$$

Definition 1.7. For an oriented combed 3-manifold $\mathfrak{C}=(M, X)$, the block number $B l^{*}(\mathfrak{C})$ is defined by

$$
\begin{aligned}
B l^{*}(\mathfrak{C})=\min \{b l(\Delta) \mid \Delta & \text { is a DS-diagram with E-cycle } \\
& \text { such that } \mathfrak{C}(\Delta) \text { is equivalent to } \mathfrak{C}\} .
\end{aligned}
$$


Obviously $B l(M)$ and $B l^{*}(\mathfrak{C})$ are topological invariants for closed orientable 3-manifolds and oriented combed 3-manifolds respectively. It is also evident that $B l(M) \leq B l^{*}(\mathfrak{C})$ for any oriented combed 3-manifold $\mathfrak{C}$ defined on an orientable closed 3-manifold $M$, and

$$
B l(M)=\min \left\{B l^{*}(\mathfrak{C}) \mid \mathfrak{C} \text { is an oriented combed 3-manifold on } M\right\} .
$$

\section{Basic properties of block numbers}

2.1. Block numbers for $M=S^{2} \times S^{1}, S^{3}$. First we will show the block numbers of the two basic 3-manifolds $S^{2} \times S^{1}$ and $S^{3}$ are given by the following theorems.

Theorem 2.1. $B l(M)=0$ if and only if $M$ is homeomorphic to $S^{2} \times S^{1}$.

Theorem 2.2. $B l\left(S^{3}\right)=1$.

Proof of Theorem 2.1. The assumption $B l(M)=0$ implies that $M$ is represented by a DS-diagram $\Delta=(G, f, P: e)$ such that $V(P)=\emptyset$. On the other hand, the only DS-diagram with E-cycle having no vertex is given by Figure 1.2. Hence, to prove the theorem, it is sufficient to show that $S^{2} \times S^{1}$ has a simple spine which yields the DS-diagram in Figure 1.2.

Let $F \equiv S^{2} \times\{*\}$ be a non-separating sphere in $S^{2} \times S^{1}$, and let $D_{1}$ and $D_{2}$ be mutually disjoint 2-disks in $F$. We can take an annulus $A$ embedded in $S^{2} \times S^{1}$ so that $\partial A=\partial D_{1} \cup \partial D_{2}$ and $P_{h} \equiv\left(F-D_{2}\right) \cup A$ forms a simple spine, whose singularity $S\left(P_{h}\right)=\partial D_{1}$ is a hoop. The DS-diagram in Figure 1.2 represents $S^{2} \times S^{1}$ with the simple spine $P_{h}$. This completes the proof.

A non-singular vector field on $S^{2} \times S^{1}$ is said to be canonical if it is everywhere tangential to a fiber $\{*\} \times S^{1}$, and an oriented combed 3-manifold $\mathfrak{C}=\left(S^{2} \times S^{1}, X\right)$ is said to be canonical if $X$ is homotopic to the canonical vector field. Then we have the following theorem which refines Theorem 2.1.

Theorem 2.3. $B l^{*}(\mathfrak{C})=0$ if and only if $\mathfrak{C}$ is a canonical oriented combed manifold on $S^{2} \times S^{1}$.

Proof. We denote by $\Delta_{h}$ the DS-diagram in Figure 1.2. Then, directly from the construction of the above spine $P_{h}$, it follows that $\mathfrak{C}\left(\Delta_{h}\right)$ is canonical. Hence the condition $B l^{*}(\mathfrak{C})=0$ implies that the oriented combed 3-manifold $\mathfrak{C}$ is equivalent to $\mathfrak{C}\left(\Delta_{h}\right)$, that is, $\mathfrak{C}$ itself is a canonical oriented combed 3 -manifold on $S^{2} \times S^{1}$.

Proof of Theorem 2.2. The 3 -sphere has a simple spine $P_{a}$, called an "abalone", which has only one vertex (see [3]). The DS-diagram $\Delta_{a}$ for the abalone 
$P_{a}$ has an E-cycle, and is represented by an E-data $\mathcal{E}\left(\Delta_{a}\right)=x^{-\ell} x^{+\ell}\left(\right.$ or $\left.x^{-r} x^{+r}\right)$. Obviously $b l\left(\Delta_{a}\right)=1$, and so $B l\left(S^{3}\right) \leq 1$. This proves $B l\left(S^{3}\right)=1$, because $\operatorname{Bl}\left(S^{3}\right) \geq 1$ by Theorem 2.1 .

2.2. Moves for E-data. We introduced in [11] the moves for DS-diagrams with E-cycle, which generate the deformations of DS-diagrams with E-cycle preserving the represented manifold (or combed manifold). Here we will review those moves in terms of the coded sequences for E-data.

Definition 2.1 (The first regular move $R_{1}$ ). Let $\Delta$ be a DS-diagram with E-cycle whose E-data $\mathcal{E}(\Delta)$ includes three subwords $W_{1}=a^{-\ell} b^{+\ell}, W_{2}=a^{+\ell} x^{+\ell}$ and $W_{3}=x^{-\ell} b^{-\ell}$. Then $R_{1}$ is defined to be a deformation changing $\Delta$ into $\Delta^{\prime}$ with the E-data $\mathcal{E}\left(\Delta^{\prime}\right)$ in which the subwords $W_{k}(k=1,2,3)$ are replaced by $W_{1}^{\prime}=b^{+\ell} a^{-\ell}, W_{2}^{\prime}=a^{+\ell}$ and $W_{3}^{\prime}=b^{-\ell}$ respectively. By this move, $a^{-\ell}$ and $b^{+\ell}$ mutually exchange their places in the coded sequence, and $x^{ \pm \ell}$ are eliminated. Hence, for the spines $P$ represented by $\Delta$ and $P^{\prime}$ represented by $\Delta^{\prime}$, we have $\# V(P)=\# V\left(P^{\prime}\right)+1$.

The alteration of the simple spine caused by the move $R_{1}$ is shown in Figure 2.1, and we can see that a formal E-data $\mathcal{E}$, namely a coded sequence, is realizable if and only if $R_{1}(\mathcal{E})$, an E-data obtained by formally applying the move $R_{1}$, is realizable. Hence we can define the inverse move $R_{1}^{-1}$ which replaces the subword $W_{1}^{\prime}=b^{+\ell} a^{-\ell}, W_{2}^{\prime}=a^{+\ell}$ and $W_{3}^{\prime}=b^{-\ell}$ by $W_{1}=a^{-\ell} b^{+\ell}, W_{2}=a^{+\ell} x^{+\ell}$ and $W_{3}=x^{-\ell} b^{-\ell}$ respectively, and we can treat the move $R_{1}$ and its inverse $R_{1}^{-1}$ quite formally.
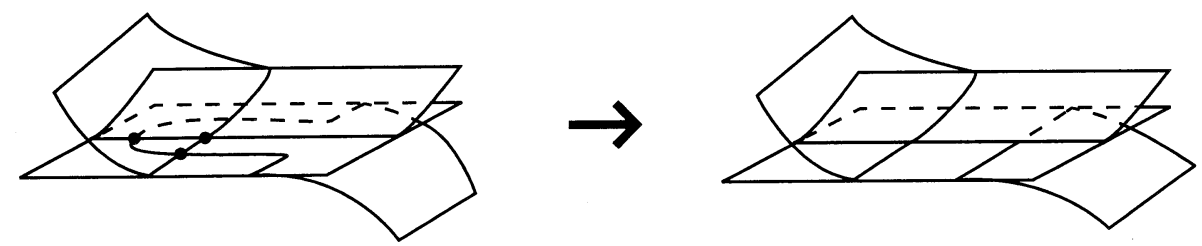

Figure 2.1. The first regular move $R_{1}$
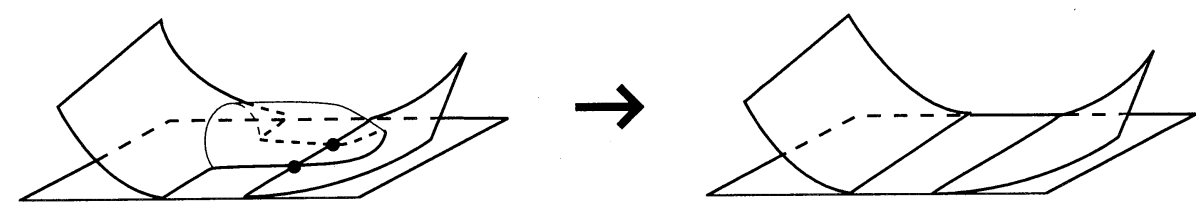

Figure 2.2. The second regular move $R_{2}$ 
Definition 2.2 (The second regular move $R_{2}$ ). Let $\Delta$ be a DS-diagram with E-cycle whose E-data $\mathcal{E}(\Delta)$ includes two subwords $W_{1}=x^{-\ell} y^{-r}$ (or $y^{-r} x^{-\ell}$ ) and $W_{2}=x^{+\ell} y^{+r}$ (or $y^{+r} x^{+\ell}$ ). Then $R_{2}$ is defined to be a deformation eliminating these two subwords $W_{1}$ and $W_{2}$. Hence, for two spines $P$ represented by $\Delta$ and $P^{\prime}$ represented by $\Delta^{\prime}=R_{2}(\Delta)$ obtained by the move $R_{2}$, we have $\# V(P)=$ $\# V\left(P^{\prime}\right)+2$.

The alteration of the simple spine by a second regular move $R_{2}$ is shown in Figure 2.2. If a formal E-data $\mathcal{E}$ is realizable, then so is $R_{2}(\mathcal{E})$. However, even if $\mathcal{E}$ is realizable, the formal E-data $R_{2}^{-1}(\mathcal{E})$ may not be realizable. Hence we must check the realizability of E-data when we apply the inverse move $R_{2}^{-1}$. In this paper, we do not use the inverse move $R_{2}^{-1}$.

If there is a sequence $\Delta=\Delta_{0}, \Delta_{1}, \Delta_{2}, \ldots, \Delta_{n}=\Delta^{\prime}$ of DS-diagrams with E-cycle such that $\Delta_{k}$ is obtained from $\Delta_{k-1}$ by one of the moves $R_{1}^{ \pm 1}$ and $R_{2}^{ \pm 1}$, then $\Delta^{\prime}$ is said to be regularly equivalent to $\Delta$, and denoted by $\Delta^{\prime} \cong \Delta$.

The following theorem was shown in [11].

Theorem 2.4. Let $\Delta$ and $\Delta^{\prime}$ be $D S$-diagrams with E-cycle. Then the oriented combed 3-manifolds $\mathfrak{C}(\Delta)$ and $\mathfrak{C}\left(\Delta^{\prime}\right)$ are mutually equivalent if and only if $\Delta \cong$ $\Delta^{\prime}$.

One more move, called the surgery move, is defined as follows. We do not use this move in this paper.

Definition 2.3 (The surgery move $S$ ). If $\Delta$ is a DS-diagram with E-cycle whose E-data $\mathfrak{E}(\Delta)$ includes a subword $W=x^{-\ell} y^{+\ell} z^{+\ell} z^{-\ell} w^{-r} y^{-\ell} w^{+r} x^{+\ell}$, then the surgery move $S$ gives a DS-diagram $\Delta^{\prime}=S(\Delta)$ with the E-data $\mathcal{E}\left(\Delta^{\prime}\right)$ obtained from $\mathfrak{E}(\Delta)$ by eliminating the subword $W$.

If there is a sequence $\Delta=\Delta_{0}, \Delta_{1}, \Delta_{2}, \ldots, \Delta_{n}=\Delta^{\prime}$ of DS-diagrams with E-cycle such that $\Delta_{k}$ is obtained from $\Delta_{k-1}$ by one of the moves $R_{1}^{ \pm 1}, R_{2}^{ \pm 1}$ and $S^{ \pm 1}$, then $\Delta^{\prime}$ is said to be equivalent to $\Delta$, and denoted by $\Delta^{\prime} \sim \Delta$.

Also the following theorem was proved in [11].

TheOREM 2.5. Let $\Delta$ and $\Delta^{\prime}$ be DS-diagrams with E-cycle. Then there is an orientation preserving homeomorphism from $M(\Delta)$ onto $M\left(\Delta^{\prime}\right)$ if and only if $\Delta \sim \Delta^{\prime}$.

According to the above two theorems, we have the following alternative definition of block number of an oriented combed 3-manifold or an orientable 3-manifold represented by a DS-diagram $\Delta$ with E-cycle:

$$
\begin{aligned}
& B l^{*}(\mathfrak{C}(\Delta))=\min \left\{b l\left(\Delta^{\prime}\right) \mid \Delta^{\prime} \cong \Delta\right\} \\
& B l(M(\Delta))=\min \left\{b l\left(\Delta^{\prime}\right) \mid \Delta^{\prime} \sim \Delta\right\}
\end{aligned}
$$


2.3. The block number and the Heegaard genus. The block number is closely related to the Heegaard genus, as is shown in the following theorem.

Theorem 2.6. $H G(M) \leq B l(M)$ for any closed orientable 3-manifold $M$ except for $S^{2} \times S^{1}$.

REMARK 2.1. The reason why the manifold $S^{2} \times S^{1}$ is exceptional is that it has a simple spine without any vertex (the DS-diagram $\Delta_{h}$ in Figure 1.2). Also the lens space $L(3,1)$ admits a simple spine with no vertex, but the DS-diagram for this simple spine has no E-cycle.

Proof. In order to prove this theorem, it is sufficient to show that, given a DS-diagram $\Delta=(G, f, P ; e)$ with $V(P) \neq \emptyset$, we can find a Heegaard splitting of $M(\Delta)$ with genus $g=b l(\Delta)$.

Let $\Sigma^{+} \subset S^{2}$ be the positive region, and $D \subset \Sigma^{+}$be the disk $D=\operatorname{cl}\left(\Sigma^{+}-\right.$ $N\left(e, S^{2}\right)$ ), where $N\left(e, S^{2}\right)$ denotes a regular neighborhood of the E-cycle $e$ in the sphere $S^{2}$. By the definition of an E-cycle $e, f(D)$ is a disk embedded in $M=B^{3} / f$. We may assume that each component of $\operatorname{cl}\left(G \cap N\left(e, S^{2}\right) \cap \Sigma^{+}\right) \subset S^{2}$ is an arc such that one of the end points is on $\partial D$, and the other is on the E-cycle $e$, that is, $c l\left(G \cap N\left(e, S^{2}\right) \cap \Sigma^{+}\right)$consists of $\nu \equiv \# V(P)$ arcs, which we denote by $\Xi=\left\{\xi_{1}, \xi_{2}, \ldots, \xi_{\nu}\right\}$. Therefore $H_{1} \equiv N(f(D) \cup f(\Xi))(N(X)$ is a regular neighborhood of $X \subset M)$ is a handle body obtained by attaching 1-handles $N\left(f\left(\xi_{i}\right)\right)$ $(i=1,2, \ldots, \nu)$ to a 3 -ball $N(f(D))$.

On the other hand, the set $\left(\Sigma^{+}-\left(D \cup N\left(G, S^{2}\right)\right)\right)$ consists of 2-disks $\psi_{i}$ $(i=1,2, \ldots, \nu)$, and so the complementary space $H_{2} \equiv \operatorname{cl}\left(M-H_{1}\right)$ can be viewed as a space obtained by attaching 1-handles $N\left(f\left(\psi_{j}\right)\right)$ to a 3-ball $c l(M-N(P))$. Therefore $\left(H_{1}, H_{2}\right)$ gives a Heegaard splitting of $M$ with genus $\nu$. We will show that, starting with this Heegaard splitting, we can get a Heegaard splitting of genus $b l(\Delta)$ by cancelling 1-handles of $H_{1}$ and $H_{2}$.

Let the arrangement $\mathcal{A}(\Delta)$ has $b l(\Delta)$ positive blocks $W_{k}^{+}(1 \leq k \leq b l(\Delta)$, and let each block $W_{k}^{+}$contains $s_{k}$ well-ordered vertices $v_{k, j}^{+}\left(1 \leq j \leq s_{k}\right)$. The arc in $\Xi$ having $v_{k, j}^{+}$as its end point is denoted by $\xi_{k, j}$, and the meridian disk of the 1-handle $N\left(f\left(\xi_{k, j}\right)\right)$ is denoted by $\delta_{k, j}$. Then the set of these disks $\delta_{k, j}$ $\left(1 \leq k \leq b l(\Delta), 1 \leq j \leq s_{k}\right)$ forms a complete meridian disk system of the handle body $H_{1}$.

On the other hand, the set of the above defined disks $\Psi \equiv\left\{f\left(\psi_{i}\right) \mid 1 \leq i \leq \nu\right\}$ gives a complete meridian disk system of the second handle body $H_{2}$. Among these disks, there is a disk $f\left(\psi_{k, j}\right)\left(1 \leq k \leq b l(\Delta), 1 \leq j \leq s_{k}-1\right)$ which intersects each of $\delta_{k, j}$ and $\delta_{k, j+1}$ at exactly one point, and does not intersect any other meridian disks $\delta_{k^{\prime}, j^{\prime}}$ of $H_{1}$ (see Figure 2.3). The system $\Psi$ contain the other $(\nu-b l(\Delta))$ meridian disks, each of which is the form $f\left(\psi_{i}\right)$ for a disk $\psi_{i} \subset \Sigma^{+}$adjacent to a negative block, and intersects with the meridian disks $\delta_{k, j}$ at least three times. 


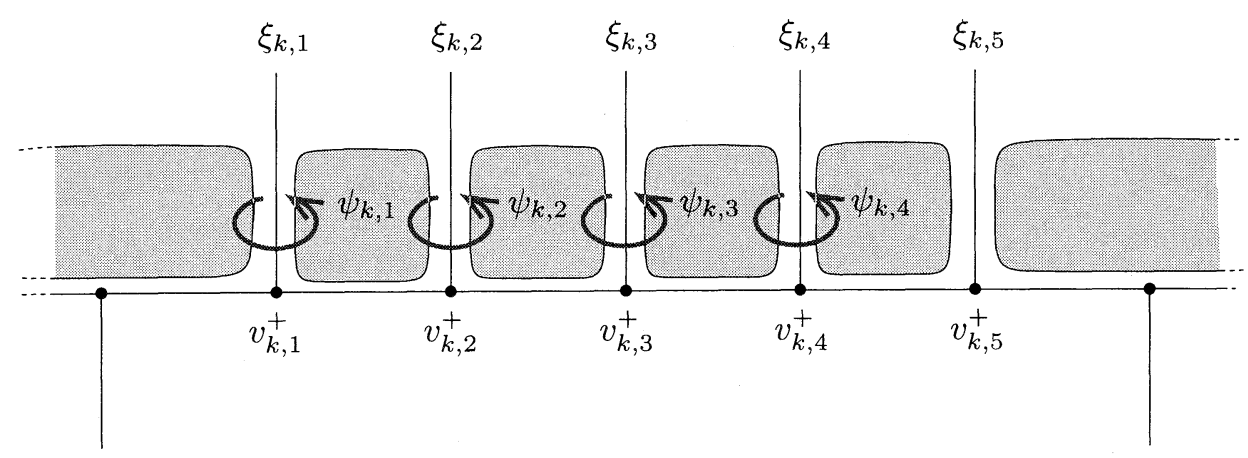

Figure 2.3. Handle cancelling

Consequently we get $(\nu-b l(\Delta))$ independent cancelling pairs $\left(\delta_{k, j}, f\left(\psi_{k, j}\right)\right)(1 \leq$ $\left.k \leq b l(\Delta), 1 \leq j \leq s_{k}-1\right)$ of meridian disks, and so the Heegaard splitting $\left(H_{1}, H_{2}\right)$ can be reduced to a Heegaard splitting of genus $b l(\Delta)$.

\section{Block numbers of lens spaces}

In this section, we treat with the lens spaces $L(p, q)$ ( $p$ and $q$ are coprime integers with $1 \leq q<p)$, and oriented combed 3-manifolds on $L(p, q)$. The 3 sphere $S^{3}$ is thought to be $L(1,1)$.

3.1. Block number of a lens space. First we will show that

THEOREM 3.1. A closed orientable 3-manifold $M$ satisfies $B l(M)=1$ if and only if $M$ is homeomorphic to the 3-sphere $S^{3}$ or a lens space $L(p, q)$.

Proof. Let $\Delta_{p, q}$ be the DS-diagram whose spine $P$ has $p$ vertices $V(P)=$ $\left\{v_{1}, v_{2}, \ldots, v_{p}\right\}$ with the codes $\phi\left(v_{k}\right)=r$ for any $k$, and whose E-data has the arrangement

$$
\begin{aligned}
& \mathcal{A}\left(\Delta_{p, q}\right)=W_{1}^{+} W_{2}^{-} \\
& W_{1}^{+}=v_{p}^{+} v_{p-1}^{+} \cdots \cdots v_{3}^{+} v_{2}^{+} v_{1}^{+}, \quad W_{2}^{-}=v_{q}^{-} v_{2 q}^{-} v_{3 q}^{-} \cdots \cdots v_{(p-1) q}^{-} v_{p q}^{-}
\end{aligned}
$$

where we use the convention: $v_{j}^{-}=v_{k}^{-}$for $j \equiv k(\bmod p)$. The graph $G \subset S^{2}$ for the DS-diagram $\Delta_{p, q}$ is shown in Figure 3.1. Obviouly $b l\left(\Delta_{p, q}\right)=1$ for any $(p, q)$, and especially $\Delta_{1,1}$ gives the DS-diagram for the abalone (see $\S 2.1$ ). It is known that the manifold $M\left(\Delta_{p, q}\right)$ is the lens space $L(p, q)$ (see [9] and [13]). This, together with Theorems 2.1, 2.2 and 2.6, shows that $B l(M)=1$ if and only if $M$ is $S^{3}$ or $L(p, q)$. 


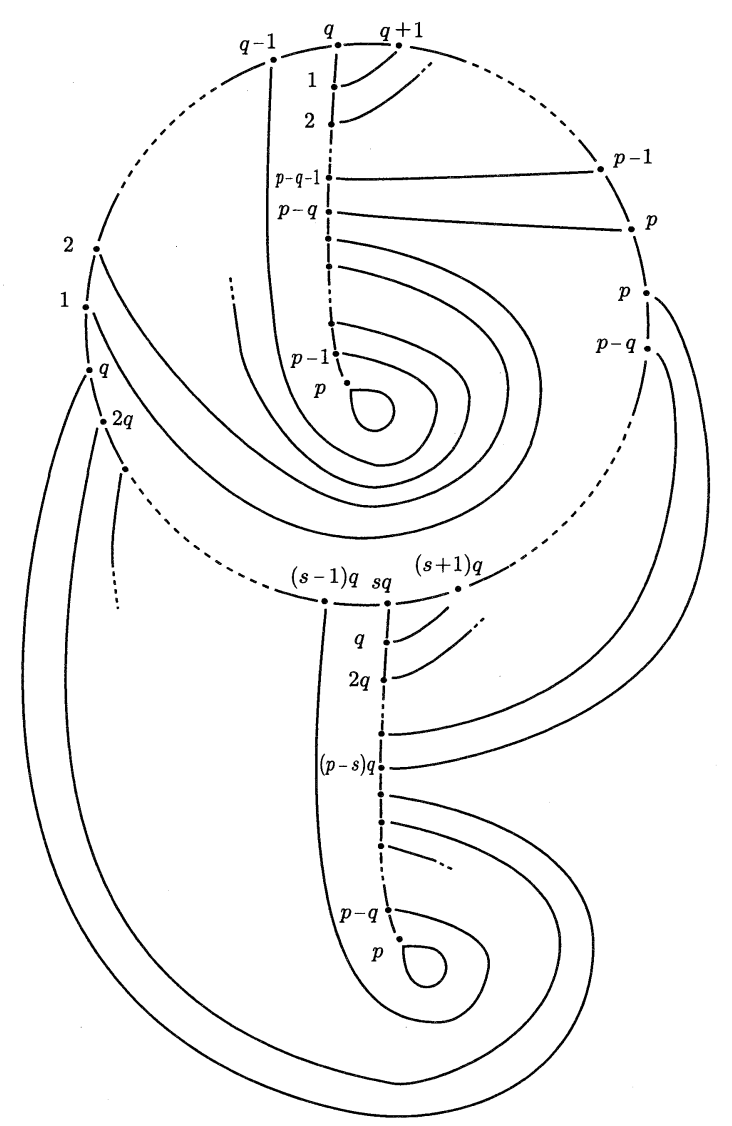

Figure 3.1. DS-diagram $\Delta_{p, q}(s q \equiv 1(\bmod p))$

3.2. Seifert fibered spaces. We will consider the relation between the Seifert fibered structure on $L(p, q)$ and the block number $B l^{*}(\mathfrak{C})$ of an oriented combed 3-manifold $\mathfrak{C}$ on $L(p, q)$. Moreover, in the next section, we will investigate the block numbers of Seifert fibered spaces on $S^{2}$ having three exceptional fibers. For this purpose, we will fix the representations for Seifert fibered spaces on the 2sphere $S^{2}$. A lens space is a Seifert fibered space on $S^{2}$ with at most two exceptional fibers.

A $(\beta / \alpha)$-fibered solid torus $V(\alpha, \beta)$ is a solid torus

$$
V(\alpha, \beta)=\left\{\left(r e^{2 \pi i \theta}, e^{2 \pi i t}\right) \mid 0 \leq r \leq 1 \text { and } \theta, t \in \mathbb{R}\right\}
$$


given a Seifert fibered structure such that a fiber is of the form

$$
\left\{\left(r e^{2 \pi i(\theta+\beta s)}, e^{2 \pi i(t+\alpha s)}\right) \mid s \in \mathbb{R}\right\}
$$

where $\alpha$ and $\beta$ are coprime integers. The map $h_{\alpha, \beta}: \partial V(\alpha, \beta) \longrightarrow \partial V(1,0)$ is defined by $h_{\alpha, \beta}\left(e^{2 \pi i(\theta+\beta s)}, e^{2 \pi i(t+\alpha s)}\right)=\left(e^{2 \pi i \theta}, e^{2 \pi i(t+s)}\right)$, which preserves the fibers.

The product space $S^{2} \times S^{1}$ is a fibered space with fibers $\{*\} \times S^{1}$, and each fiber has a fibered neighborhood of type $V(1,0)$. Given $n$ pairs $\left(\alpha_{k}, \beta_{k}\right)(1 \leq k \leq n)$ of coprime integers with $1 \leq \beta_{k}<\alpha_{k}$, we define a Seifert fibered space $\Sigma\left(n ;\left(\alpha_{k}, \beta_{k}\right)\right)$, which has $S^{2}$ as its base space and has $n$ exceptional fibers, by

$$
\begin{aligned}
\Sigma\left(n ;\left(\alpha_{k}, \beta_{k}\right)\right)= & \left(S^{2} \times S^{1}-\left(V_{1} \cup V_{2} \cup \cdots \cup V_{n}\right)\right) \\
& \cup V\left(\alpha_{1}, \beta_{1}\right) \cup V\left(\alpha_{2}, \beta_{2}\right) \cup \cdots \cup V\left(\alpha_{n}, \beta_{n}\right),
\end{aligned}
$$

where each $V_{k}$ is a fibered neighborhood of type $V(1,0)$, and the fibered solid torus $V\left(\alpha_{k}, \beta_{k}\right)$ is glued back to $V_{k}$ by the map $h_{\alpha_{k}, \beta_{k}}$.

In general, a non-singular vector field $X_{0}$ on a Seifert fibered space $M$ is said to be canonical if $X_{0}$ is everywhere tangential to a fiber. An oriented combed 3manifold $\mathfrak{C}=(M, X)$ on an oriented Seifert fibered space $M$ is said to be canonical if it belongs to the same equivalence class as $\mathfrak{C}_{0}=\left(M, X_{0}\right)$ for a canonical vector field $X_{0}$. If $\mathfrak{C}_{0}=\left(M, X_{0}\right)$ is canonical, then $\mathfrak{C}_{0}^{*}=\left(M,-X_{0}\right)$ is also canonical. Hence, a Seifert fibered structure on an oriented 3-manifold $M$ determines two equivalence classes of oriented combed 3 -manifolds, maybe $\mathfrak{C}_{0}$ and $\mathfrak{C}_{0}^{*}$ are mutually equivalent.

3.3. Canonical oriented combed 3-manifold on $L(p, q)$. A lens space $L(p, q)$ has a Seifert fibered structures $\Sigma(1 ;(p, q))$ which has only one exceptional fiber. It is known that the oriented combed 3-manifold determined by each of the DS-diagrams $\Delta_{p, q}$ and its conjugate $\Delta_{p, q}^{*}$ gives the canonical oriented combed 3manifold on $L(p, q)$ equipped with the Seifert fibered structure $\Sigma(1 ;(p, \dot{q}))$ (see [9] and [13]). Especially the abalone $\Delta_{a}=\Delta_{1,1}$ gives the canonical oriented combed 3-manifold on $S^{3}$ equipped with the Hopf fibering, which has no exceptional fiber.

Besides the above structure, a lens space $L(p, q)$ has Seifert fibered structures $\Sigma\left(2 ;\left(\alpha_{k}, \beta_{k}\right)\right)$ for adequate choices of pairs $\left(\alpha_{1}, \beta_{1}\right)$ and $\left(\alpha_{2}, \beta_{2}\right)$ which has two exceptional fibers. Also the product manifold $S^{2} \times S^{1}$ has the Seifert fibered structure of type $\Sigma\left(2 ;\left(\alpha_{k}, \beta_{k}\right)\right)$. Hence, on a Seifert fibered space $M=L(p, q)$ or $S^{2} \times S^{1}$, there are several canonical vector fields, which are corresponding to each Seifert fibered structure on $M$. However we can show the following lemma, whose proof will be given in the next section after some more definitions.

Lemma 3.2. Let $M$ be an orientable 3-manifold equipped with a Seifert fibered structure $\Sigma\left(2 ;\left(\alpha_{k}, \beta_{k}\right)\right)$, and let $\Delta$ be a DS-diagram with E-cycle such that the oriented combed 3-manifold $\mathfrak{C}(\Delta)$ on $M$ is canonical for this Seifert fibered struc- 
ture. Then $\Delta$ is regularly equivalent to a DS-diagram $\Delta^{\prime}$ satisfying bl $\left(\Delta^{\prime}\right)=1$.

Moreover we can prove the following lemma.

Lemma 3.3. If a DS-diagram $\Delta$ with E-cycle satisfies that $b l(\Delta)=1$ and $B l(M(\Delta)) \neq 0$, then $\Delta$ is regularly equivalent to $\Delta_{p, q}$ or $\Delta_{p, q}^{*}$ for some $(p, q)$. And if $b l(\Delta)=1$ and $B l(M(\Delta))=0$, namely $M(\Delta)=S^{2} \times S^{1}$, then $\Delta$ is regularly equivalent to $\Delta_{h}$.

By the above two lemmas, we have that there are only two equivalence classes, $\left[\mathfrak{C}\left(\Delta_{p, q}\right)\right]$ and $\left[\mathfrak{C}\left(\Delta_{p, q}^{*}\right)\right]$, of canonical oriented combed 3-manifolds on $L(p, q)$, and that there are only one class $\mathfrak{C}\left(\Delta_{h}\right)$ of canonical oriented combed 3-manifolds on $S^{2} \times S^{1}$. Hence a "canonical" oriented combed manifold on $M=L(p, q)$ or $S^{2} \times S^{1}$ makes sense, even if we do not specify the Seifert fibered structure on $M$, and we have that

Theorem 3.4. An oriented combed manifold $\mathfrak{C}$ satisfies $B l(\mathfrak{C})=0$ if and only if $\mathfrak{C}$ is defined on $S^{2} \times S^{1}$ and is canonical. And $B l(\mathfrak{C})=1$ if and only if $\mathfrak{C}$ is a canonical oriented combed 3 -manifold on some lens space $L(p, q)$, including $S^{3}=L(1,1)$.

Proof of Lemma 3.3. Let $\Delta=(G, f, P ; e)$ be a DS-diagram with E-cycle such that $b l(\Delta)=1$, and let the spine $P$ have $n$ vertices $V(P)=\left\{v_{1}, v_{2}, \ldots, v_{n}\right\}$. Then we may assume that the E-data $\mathcal{E}(\Delta)=(\phi, \mathcal{A}(\Delta))$ has the arrangement $\mathcal{A}(\Delta)=W_{1}^{+} W_{2}^{-}$for a positive subword $W_{1}^{+}=v_{1}^{+} v_{2}^{+} \cdots v_{n}^{+}$and a negative subword $W_{2}^{-}=v_{k_{1}}^{-} v_{k_{2}}^{-} \cdots v_{k_{n}}^{-}$, where $\left\{k_{1}, k_{2}, \ldots, k_{n}\right\}$ is a permutation of $\{1,2, \ldots, n\}$.

A method for reconstructing the DS-diagram from an E-data is exhibited in [8]. Using this method, we can see that if $b l(\Delta)=1$ and $\phi\left(v_{i}\right) \neq \phi\left(v_{j}\right)$ for some $v_{i}$ and $v_{j}$, then we can find a vertex $v_{k} \in V(P)$ such that $\phi\left(v_{k}\right) \neq \phi\left(v_{k+1}\right)$ and $v_{k}^{-}$is adjacent to $v_{k+1}^{-}$within the negative block $W_{2}^{-}$. Hence, if $\phi\left(v_{i}\right) \neq \phi\left(v_{j}\right)$ for some $v_{i}$ and $v_{j}$, we can apply the second regular move $R_{2}$ and decrease the number of vertices. Repeating the applications of $R_{2}$ as many times as possible, we can get a DS-diagram $\Delta^{\prime}=\left(G^{\prime}, f^{\prime}, P^{\prime} ; e^{\prime}\right)$ such that $b l\left(\Delta^{\prime}\right)=0$ or 1 , and $\phi(v)=\phi\left(v^{\prime}\right)$ for any $v, v^{\prime} \in V\left(P^{\prime}\right)$. If $b l\left(\Delta^{\prime}\right)=0$, then obviously $\Delta^{\prime}=\Delta_{h}$. Hence we may assume that the code $\phi$ of the E-data $\mathcal{E}(\Delta)$ satisfies $\phi\left(v_{i}\right)=\phi\left(v_{j}\right)$ for any $v_{i}, v_{j} \in V(P)$.

Also by the above mentioned method in [8], we can decide the realizability of a formal E-data. By this method, we have that a formal E-data

$$
\mathcal{E}_{f}=u_{1}^{+r} u_{2}^{+r} \cdots u_{p}^{+r} u_{k_{1}}^{-r} u_{k_{2}}^{-r} \cdots u_{k_{p}}^{-r} \quad \text { or } \quad u_{1}^{+\ell} u_{2}^{+\ell} \cdots u_{p}^{+\ell} u_{k_{1}}^{-\ell} u_{k_{2}}^{-\ell} \cdots u_{k_{p}}^{-\ell}
$$

is realizable if and only if $\mathcal{E}_{f}$ is one of $\mathcal{E}\left(\Delta_{p, q}\right)$ or $\mathcal{E}\left(\Delta_{p, q}^{*}\right)$. This completes the proof of Lemma 3.3. 


\section{Block numbers for Seifert fibered spaces}

In this section, we will consider the Seifert fibered space $M=\Sigma\left(3 ;\left(\alpha_{k}, \beta_{k}\right)\right)$ which has the 2-sphere $S^{2}$ as its base manifold and three exceptional fibers with the indices $\left(\alpha_{k}, \beta_{k}\right)(k=1,2,3)$, and we will show that

Theorem 4.1. The canonical oriented combed 3-manifold $\mathfrak{C}$ on $M=$ $\Sigma\left(3 ;\left(\alpha_{k}, \beta_{k}\right)\right)$ satisfies $B l^{*}(\mathfrak{C})=B l(M)=H G(M)=2$.

Also the proof of Lemma 3.2 will be given in this section.

4.1. A subword for the fibered solid torus $V(\alpha, \beta)$. In this section, we will give a subword $\Omega(\alpha, \beta)$ of a coded sequence which is thought to be a subword representing the fibered solid torus $V(\alpha, \beta)$ (cf. §3.2). And, using these subwords, we will give E-data which represent the canonical oriented combed 3-manifolds on the Seifert fibered spaces $\Sigma\left(2 ;\left(\alpha_{k}, \beta_{k}\right)\right)$ and $\Sigma\left(3 ;\left(\alpha_{k}, \beta_{k}\right)\right)$.

The subword $\Omega(\alpha, \beta)$ is defined by the continued fraction for $\beta / \alpha$, and so we begin with the notation for continued fractions. We use the notation $\left[k_{1}, k_{2}, k_{3}, \ldots, k_{n}\right]$ in order to represent a finite continued fraction:

$$
\left[k_{1}, k_{2}, k_{3}, \ldots, k_{n}\right]=\frac{1}{k_{1}+\frac{1}{k_{2}+\frac{1}{k_{3}+\ddots}}}
$$

For coprime integers $\alpha$ and $\beta$ such that $1 \leq \beta \leq \alpha / 2$, the ratio $\beta / \alpha$ can be uniquely expanded to a continued fraction $\beta / \alpha=\left[k_{1}, k_{2}, \ldots, k_{n}\right]$ with $k_{j} \geq 2$ $(1 \leq j \leq n)$. We denote by $\lambda \equiv \lambda(\alpha, \beta)$ the integer $\left(\sum k_{j}\right)-2$, which is called the size of $(\alpha, \beta)$. The subwords $\Omega(\alpha, \beta)$ for coprime integers $\alpha, \beta$ with $1 \leq \beta \leq \alpha / 2$ are inductively defined as follows.

Definition 4.1. The subword $\Omega(\alpha, \beta)$, which consists of $2 \lambda$ symbols $x_{j}^{-\sigma}$ and $x_{j}^{+\sigma}\left(\sigma=\phi\left(x_{j}\right)\right.$ (the code $\ell$ or $r$ ) and $\left.j=1, \ldots, \lambda\right)$, is defined by

(0) $\Omega(2,1)=\emptyset$ (the empty word),

(1) $\Omega(3,1)=x_{1}^{+r} x_{1}^{-r}$,

(2) if $n$ is odd, $\beta / \alpha=\left[k_{1}, k_{2}, \ldots, k_{n}\right], k_{n} \geq 3$ and $\beta^{\prime} / \alpha^{\prime}=\left[k_{1}, \ldots, k_{n-1}, k_{n}-1\right]$, then $\Omega(\alpha, \beta)=x_{\lambda}^{+r} \Omega\left(\alpha^{\prime}, \beta^{\prime}\right) x_{\lambda}^{-r}$,

(3) if $n$ is even, $\beta / \alpha=\left[k_{1}, k_{2}, \ldots, k_{n}\right], k_{n} \geq 3$ and $\beta^{\prime} / \alpha^{\prime}=\left[k_{1}, \ldots, k_{n-1}, k_{n}-1\right]$, then $\Omega(\alpha, \beta)=x_{\lambda}^{-\ell} \Omega\left(\alpha^{\prime}, \beta^{\prime}\right) x_{\lambda}^{+\ell}$, 
(4) if $n$ is even, $\beta / \alpha=\left[k_{1}, k_{2}, \ldots, k_{n-1}, 2\right]$ and $\beta^{\prime} / \alpha^{\prime}=\left[k_{1}, k_{2}, \ldots, k_{n-1}+1\right]$, then $\Omega(\alpha, \beta)=x_{\lambda}^{-\ell} \Omega\left(\alpha^{\prime}, \beta^{\prime}\right) x_{\lambda}^{+\ell}$,

(5) if $n$ is odd, $\beta / \alpha=\left[k_{1}, k_{2}, \ldots, k_{n-1}, 2\right]$ and $\beta^{\prime} / \alpha^{\prime}=\left[k_{1}, k_{2}, \ldots, k_{n-1}+1\right]$, then $\Omega(\alpha, \beta)=x_{\lambda}^{+r} \Omega\left(\alpha^{\prime}, \beta^{\prime}\right) x_{\lambda}^{-r}$.

Noticing that $\left[a_{1}, a_{2}, \ldots, a_{n}, 1\right]=\left[a_{1}, a_{2}, \ldots, a_{n}+1\right]$ in general, we can see that the above definition determines $\Omega(\alpha, \beta)$ for any $(\alpha, \beta)$ with $1 \leq \beta \leq \alpha / 2$. In order to specify the letter " $x$ " used for $\Omega(\alpha, \beta)$, we represent it as $\Omega(\alpha, \beta ; x)$; for example,

$$
\Omega(12,5 ; y)=y_{4}^{+r} y_{3}^{-\ell} y_{2}^{-\ell} y_{1}^{+r} y_{1}^{-r} y_{2}^{+\ell} y_{3}^{+\ell} y_{4}^{-r} \quad(5 / 12=[2,2,2]) .
$$

By $\Omega^{*}(\alpha, \beta)$ we denote the subword obtained from $\Omega(\alpha, \beta)$ by the substitutions of signatures and codes: $+\longrightarrow-,-\longrightarrow+, r \longrightarrow \ell, \quad \ell \longrightarrow r$. And, for coprime integers $\alpha, \beta$ with $\alpha / 2<\beta<\alpha$, we define $\Omega(\alpha, \beta)$ to be $\Omega(\alpha, \beta)=\Omega^{*}(\alpha, \alpha-\beta)$. The following two theorems are shown in [13].

TheOREM 4.2. There exists a DS-diagram $\Delta$ having the E-data

$$
\mathcal{E}(\Delta)=\Omega\left(\alpha_{1}, \beta_{1} ; x\right) \Omega\left(\alpha_{2}, \beta_{2} ; y\right),
$$

and the represented manifold $M(\Delta)$ admits a Seifert fibered structure $\Sigma\left(2 ;\left(\alpha_{k}, \beta_{k}\right)\right)$. And moreover the oriented combed 3-manifold $\mathfrak{C}(\Delta)$ is canonical with respect to this Seifert fibered structure.

TheOREM 4.3. There exists a DS-diagram $\Delta$ represented by the E-data

$$
\begin{aligned}
& \mathcal{E}(\Delta) \\
& =\left(a_{1}^{-\ell} a_{3}^{+\ell}\right) \Omega\left(\alpha_{1}, \beta_{1} ; x\right)\left(a_{2}^{-\ell} a_{1}^{+\ell}\right) \Omega\left(\alpha_{2}, \beta_{2} ; y\right)\left(a_{3}^{-\ell} a_{2}^{+\ell}\right) \Omega\left(\alpha_{3}, \beta_{3} ; z\right),
\end{aligned}
$$

and the oriented combed 3-manifold $\mathfrak{C}(\Delta)$ is a canonical one on the Seifert fibered space $M(\Delta)=\Sigma\left(3 ;\left(\alpha_{k}, \beta_{k}\right)\right)$.

We can reconfirm these theorems by means of the method in [10], in which we have exhibited how we can recognize the Seifert fibered structure by a DS-diagram.

4.2. Some more moves. The E-data in the formulas (4.1), (4.2) have too many blocks. Hence, in order to prove Lemma 3.2 and Theorem 4.1, we must reduce the block number. For this purpose, we will prepare in this subsection some more moves which are defined as some compositions of regular moves and their inverses, and are used for decreasing the block number without altering the equivalence class of the represented combed 3-manifold.

Let $T_{\ell, \ell}^{\ell}$ be the inverse move of the first regular move $R_{1}$. In terms of the coded sequence, the move $T_{\ell, \ell}^{\ell} \equiv R_{1}^{-1}$ can be represented as the following replacements 
(i)--(iii) of subwords:

$$
T_{\ell, \ell}^{\ell}: \text { (i) } b^{+\ell} a^{-\ell} \longrightarrow a^{-\ell} b^{+\ell}, \text { (ii) } a^{+\ell} \longrightarrow a^{+\ell} x^{+\ell}, \text { (iii) } b^{-\ell} \longrightarrow x^{-\ell} b^{-\ell},
$$

where $x^{ \pm \ell}$ are new symbols appearing by the move $T_{\ell, \ell}^{\ell} \equiv R_{1}^{-1}$. Composing the regular moves $R_{1}^{ \pm 1}$ and $R_{2}^{ \pm 1}$, we can obtain seven other similar moves $T_{\sigma_{2}, \sigma_{3}}^{\sigma_{1}}\left(\sigma_{j}=\ell\right.$ or $r$ ) (cf. [11]), each of which causes the following replacement of subwords in $\mathcal{E}(\Delta)$ :
$T_{\ell, r}^{\ell}:(\mathrm{i}) b^{+r} a^{-\ell} \longrightarrow a^{-\ell} b^{+r}$,
(ii) $a^{+\ell} \longrightarrow a^{+\ell} x^{+r}$
(iii) $b^{-r} \longrightarrow b^{-r} x^{-r}$
$T_{r, \ell}^{\ell}:$ (i) $b^{+\ell} a^{-r} \longrightarrow a^{-r} b^{+\ell}$,
(ii) $a^{+r} \longrightarrow x^{+r} a^{+r}$,
(iii) $b^{-\ell} \longrightarrow x^{-r} b^{-\ell}$
$T_{r, r}^{\ell}:$ (i) $b^{+r} a^{-r} \longrightarrow a^{-r} b^{+r}$,
(ii) $a^{+r} \longrightarrow x^{+\ell} a^{+r}$,
(iii) $b^{-r} \longrightarrow b^{-r} x^{-\ell}$
$T_{\ell, \ell}^{r}:$ (i) $a^{-\ell} b^{+\ell} \longrightarrow b^{+\ell} a^{-\ell}$,
(ii) $a^{+\ell} \longrightarrow a^{+\ell} x^{+r}$
(iii) $b^{-\ell} \longrightarrow x^{-r} b^{-\ell}$
$T_{\ell, r}^{r}:$ (i) $a^{-\ell} b^{+r} \longrightarrow b^{+r} a^{-\ell}$,
(ii) $a^{+\ell} \longrightarrow a^{+\ell} x^{+\ell}$
(iii) $b^{-r} \longrightarrow b^{-r} x^{-\ell}$
$T_{r, \ell}^{r}:$ (i) $a^{-r} b^{+\ell} \longrightarrow b^{+\ell} a^{-r}$,
(ii) $a^{+r} \longrightarrow x^{+r} a^{+r}$,
(iii) $b^{-\ell} \longrightarrow x^{-r} b^{-\ell}$
$T_{r, r}^{r}:(\mathrm{i}) a^{-r} b^{+r} \longrightarrow b^{+r} a^{-r}$
(ii) $a^{+r} \longrightarrow x^{+r} a^{+r}$,
(iii) $b^{-r} \longrightarrow b^{-r} x^{-r}$.

Each of the above eight moves $T_{\sigma_{2}, \sigma_{3}}^{\sigma_{1}}$ exchanges the positions of $a^{-}$and $b^{+}$in the arrangement $\mathcal{A}(\Delta)$, and so we can use them for decreasing the block number without altering the regular equivalence class.

EXAMPLE 4.1. Consider the DS-diagram $\Delta$ in Example 1.1 whose E-data is given by

$$
\mathcal{E}(\Delta)=\left\{a^{+\ell} b^{+r} c^{+\ell} b^{-r} d^{+r} d^{-r} a^{-\ell} c^{-\ell}\right\}
$$

We can apply the move $T_{r, r}^{r}$ on the subword $b^{-r} d^{+r}$ together with $b^{+r}, d^{-r}$. After this move, we get a DS-diagram $\Delta^{\prime}$ with the E-data

$$
\mathcal{E}\left(\Delta^{\prime}\right)=\left\{a^{+\ell} x^{+r} b^{+r} c^{+\ell} d^{+r} b^{-r} d^{-r} x^{-r} a^{-\ell} c^{-\ell}\right\} .
$$

Therefore we have that $\Delta^{\prime} \cong \Delta$ and $1=b l\left(\Delta^{\prime}\right)<b l(\Delta)=2$. Moreover, applying the second regular move $R_{2}$ on the subwords $a^{+\ell} x^{+r}$ and $x^{-r} a^{-\ell}$ of $\mathcal{E}\left(\Delta^{\prime}\right)$, we get a DS-diagram $\Delta^{\prime \prime}$ with the E-data

$$
\mathcal{E}\left(\Delta^{\prime \prime}\right)=\left\{b^{+r} c^{+\ell} d^{+r} b^{-r} d^{-r} c^{-\ell}\right\}
$$

and, applying $R_{2}$ once more, we finally get a DS-diagram with the E-data $\left\{b^{+r} b^{-r}\right\}$, which is the E-data for the abalone $\Delta_{a}$. Hence we can conclude that $M(\Delta)=S^{3}$.

Let $\Delta=(G, f, P ; e)$ be a DS-diagram whose E-data has an arrangement

$$
\mathcal{A}(\Delta)=U_{1}^{+} U_{2}^{-} W_{0}^{-} W_{1}^{+} W_{2}^{-} \cdots \cdots W_{2 n-1}^{+} W_{2 n}^{-} W_{2 n+1}^{+}
$$

where $U_{1}^{+}, W_{2 k-1}^{+}$are positive subwords and $U_{2}^{-}, W_{2 k}^{-}$are negative subwords. If both $W_{0}^{-}$and $W_{2 n+1}^{+}$are empty words, then (4.3) gives a blockwise representation. 
We consider the case $f\left(U_{1}^{+}\right) \cap f\left(U_{2}^{-}\right)=\emptyset$, namely if $U_{1}^{+}=b_{\nu}^{+} b_{\nu-1}^{+} \cdots b_{2}^{+} b_{1}^{+}$, then $b_{k}^{-}$does not appear in $U_{2}^{-}$for any $1 \leq k \leq \nu$. In this case, $U_{2}^{-}$can be written as $U_{2}^{-}=a_{1}^{-} a_{2}^{-} \cdots a_{\mu-1}^{-} a_{\mu}^{-}$. Then, making successive applications of the moves $T_{\sigma, \sigma^{\prime}}^{\ell}\left(\sigma=\phi\left(a_{1}\right), \sigma^{\prime}=\phi\left(b_{j}\right), j=1,2, \ldots, \nu\right)$, we obtain a DS-diagram $\Delta^{\prime}$ with an arrangement of E-data

$$
\mathcal{A}\left(\Delta^{\prime}\right)=a_{1}^{-} U_{1}^{+} \widetilde{U}_{2}^{-} \widetilde{W}_{0}^{-} \widetilde{W}_{1}^{+} \widetilde{W}_{2}^{-} \cdots \cdots \widetilde{W}_{2 n}^{-} \widetilde{W}_{2 n+1}^{+},
$$

which satisfies that

(i) $\widetilde{U}_{2}^{-}=a_{2}^{-} a_{3}^{-} \cdots a_{\mu-1}^{-} a_{\mu}^{-}$,

(ii) $\widetilde{W}_{2 k}^{+}$(or $\widetilde{W}_{2 k-1}^{-}$) is a positive (respectively negative) subword which differs from $W_{2 k}^{+}$(respectively $W_{2 k-1}^{-}$) only by the new symbols created by the moves,

(iii) $\widetilde{W}_{2 k}^{+}$includes some new symbols if and only if the symbol $a_{1}^{+}$appears in the original subword $W_{2 k}^{+}$, and

(iv) $\widetilde{W}_{2 k-1}^{-}$includes some new symbols if and only if the original subword $W_{2 k-1}^{-}$ contains the symbol $b_{j}^{-}$for some $j=1,2, \ldots, \nu$.

Further successive applications of the moves $T_{\sigma, \sigma^{\prime}}^{\ell}\left(\sigma=\phi\left(a_{i}\right), \sigma^{\prime}=\phi\left(b_{j}\right)\right.$, $1 \leq j \leq \nu$ and $2 \leq i \leq \mu$ ) lead us to the following lemma.

Lemma 4.4. Let $\Delta=(G, f, P ; e)$ be a $D S$-diagram having an arrangement of E-data of the form (4.3). If $f\left(U_{1}^{+}\right) \cap f\left(U_{2}^{-}\right)=\emptyset, U_{1}^{+}=b_{\nu}^{+} b_{\nu-1}^{+} \cdots b_{2}^{+} b_{1}^{+}$and $U_{2}^{-}=a_{1}^{-} a_{2}^{-} \cdots a_{\mu-1}^{-} a_{\mu}^{-}$, then, by adequate successive applications of the moves $T_{\sigma, \sigma^{\prime}}^{\ell}$, we can obtain a DS-diagram $\Delta^{\prime}$ such that $\Delta^{\prime} \cong \Delta$ and the E-data $\mathcal{E}\left(\Delta^{\prime}\right)$ has an arrangement

$$
\mathcal{A}\left(\Delta^{\prime}\right)=U_{2}^{-} U_{1}^{+} \widetilde{W}_{0}^{-} \widetilde{W}_{1}^{+} \widetilde{W}_{2}^{-} \cdots \cdots \widetilde{W}_{2 n}^{-} \widetilde{W}_{2 n+1}^{+},
$$

satisfying the following conditions (i)-(iii):

(i) $\widetilde{W}_{2 k}^{+}$(or $\left.\widetilde{W}_{2 k-1}^{-}\right)$is a positive (respectively negative) subword which differs from $W_{2 k}^{+}$(respectively $W_{2 k-1}^{-}$) only by the new symbols created by the moves,

(ii) $\widetilde{W}_{2 k}^{+}$includes some new symbols if and only if the symbol $a_{i}^{+}$appears in the original subword $W_{2 k}^{+}$for some $i=1,2, \ldots, \mu$,

(iii) $\widetilde{W}_{2 k-1}^{-}$includes some new symbols if and only if the original subword $W_{2 k-1}^{-}$ contains the symbol $b_{j}^{-}$for some $j=1,2, \ldots, \nu$.

By similar successive applications of $T_{\sigma, \sigma^{\prime}}^{r}$ instead of $T_{\sigma, \sigma^{\prime}}^{\ell}$, we have that

LemMA 4.5. Let $\Delta=(G, f, P ; e)$ be a DS-diagram having an arrangement of E-data of the form

$$
\mathcal{A}(\Delta)=U_{1}^{-} U_{2}^{+} W_{0}^{+} W_{1}^{-} W_{2}^{+} \cdots \cdots W_{2 n-1}^{-} W_{2 n}^{+} W_{2 n+1}^{-}
$$


If $f\left(U_{1}^{-}\right) \cap f\left(U_{2}^{+}\right)=\emptyset, U_{1}^{-}=b_{\nu}^{-} b_{\nu-1}^{-} \cdots b_{2}^{-} b_{1}^{-}$and $U_{2}^{+}=a_{1}^{+} a_{2}^{+} \cdots a_{\mu-1}^{+} a_{\mu}^{+}$, then, by adequate successive applications of the moves $T_{\sigma, \sigma^{\prime}}^{r}$, we can obtain a DS-diagram $\Delta^{\prime}$ such that $\Delta^{\prime} \cong \Delta$ and the E-data $\mathcal{E}\left(\Delta^{\prime}\right)$ has an arrangement

$$
\mathcal{A}\left(\Delta^{\prime}\right)=U_{2}^{+} U_{1}^{-} \widetilde{W}_{0}^{+} \widetilde{W}_{1}^{-} \widetilde{W}_{2}^{+} \ldots \ldots \widetilde{W}_{2 n}^{+} \widetilde{W}_{2 n+1}^{-},
$$

satisfying the following conditions (i)-(iii):

(i) $\widetilde{W}_{2 k}^{-}$(or $\widetilde{W}_{2 k-1}^{+}$) is a negative (respectively positive) subword which differs from $W_{2 k}^{-}$(respectively $W_{2 k-1}^{+}$) only by the new symbols created by the moves,

(ii) $\widetilde{W}_{2 k}^{-}$includes some new symbols if and only if the symbol $a_{i}^{-}$appears in the original subword $W_{2 k}^{-}$for some $i=1,2, \ldots, \mu$,

(iii) $\widetilde{W}_{2 k-1}^{+}$includes some new symbols if and only if the original subword $W_{2 k-1}^{+}$ contains the symbol $b_{j}^{+}$for some $j=1,2, \ldots, \nu$.

In the case where the formula (4.3) is a blockwise representation, namely $W_{0}^{-}=W_{2 n+1}^{+}=\emptyset$, the resulting arrangement (4.4) gives a blockwise representation

$$
\mathcal{A}\left(\Delta^{\prime}\right)=\left(U_{1}^{+} \widetilde{W}_{1}^{+}\right) \widetilde{W}_{2}^{-} \widetilde{W}_{3}^{+} \ldots \ldots \widetilde{W}_{2 n-1}^{+}\left(\widetilde{W}_{2 n 1}^{-} U_{2}^{-}\right),
$$

and we have $b l\left(\Delta^{\prime}\right)=b l(\Delta)-1$. Hence by Lemmas 4.4 and 4.5 we get the following lemma, which gives a sufficient condition for the block number to be decreased by regular moves.

Lemma 4.6. Let $\Delta=(G, f, P ; e)$ be a DS-diagram whose E-data has an arrangement including a positive block $U^{+}$and a negative block $V^{-}$such that $f\left(U^{+}\right) \cap f\left(V^{-}\right)=\emptyset$ and they are mutually adjacent, then there exists a DS-diagram $\Delta^{\prime}$ such that $\Delta^{\prime} \cong \Delta$ and $b l\left(\Delta^{\prime}\right)=b l(\Delta)-1$.

4.3. Applications of Lemmas 4.4 and 4.5 to a subword $\Omega(\alpha, \beta)$. Consider a DS-diagram $\Delta=(G, f, P ; e)$ whose E-data includes $\Omega(\alpha, \beta)$ as its subword, that is, $\mathcal{E}(\Delta)=\Omega(\alpha, \beta) \Phi$ for some subword $\Phi$. Let $\alpha$ and $\beta$ be coprime integers such that $1 \leq \beta \leq \alpha / 2$ and $\beta / \alpha=\left[k_{1}, k_{2}, \ldots, k_{n}\right]\left(k_{j} \geq 2\right)$. The subword $\Omega(\alpha, \beta)$ has an arrangement represented as

$$
\mathcal{A}(\Omega(\alpha, \beta))=\left(V_{2 m-1}^{-} V_{2 m-2}^{+} \cdots V_{1}^{-}\right)\left(W_{1}^{+} W_{2}^{-}\right)\left(U_{1}^{+} \cdots U_{2 m-2}^{-} U_{2 m-1}^{+}\right) \text {if } n=2 m,
$$

and

$$
\mathcal{A}(\Omega(\alpha, \beta))=\left(V_{2 m}^{+} V_{2 m-1}^{-} \cdots V_{1}^{-}\right)\left(W_{1}^{+} W_{2}^{-}\right)\left(U_{1}^{+} \cdots U_{2 m-1}^{+} U_{2 m}^{-}\right) \text {if } n=2 m+1
$$

for positive subwords $V_{2 k}^{+}, U_{2 k-1}^{+}, W_{1}^{+}$and negative subwords $V_{2 k-1}^{-}, U_{2 k}^{-}, W_{2}^{-}$ which satisfy that

$$
\begin{aligned}
& f\left(W_{1}^{+}\right)=f\left(W_{2}^{-}\right), \\
& f\left(V_{2 k-1}^{-}\right)=f\left(U_{2 k-1}^{+}\right) \text {for any } k, \text { and }
\end{aligned}
$$




$$
f\left(V_{2 k}^{+}\right)=f\left(U_{2 k}^{-}\right) \text {for any } k .
$$

When $\alpha / 2 \leq \beta<\alpha$, we have a similar representation of $\Omega(\alpha, \beta)$ such that

$$
\begin{aligned}
& \mathcal{A}(\Omega(\alpha, \beta))=\left(V_{2 m-1}^{+} V_{2 m-2}^{-} \cdots V_{1}^{+}\right)\left(W_{1}^{-} W_{2}^{+}\right)\left(U_{1}^{-} \cdots U_{2 m-1}^{-} U_{2 m}^{+}\right), \text {or } \\
& \mathcal{A}(\Omega(\alpha, \beta))=\left(V_{2 m}^{-} V_{2 m-1}^{+} \cdots V_{1}^{+}\right)\left(W_{1}^{-} W_{2}^{+}\right)\left(U_{1}^{-} \cdots U_{2 m-1}^{-} U_{2 m}^{+}\right) .
\end{aligned}
$$

In what follows, we consider only the case where $1 \leq \beta \leq \alpha / 2$ and $n=2 m+1$. Other cases can be treated quite similarly. Since $f\left(U_{1}^{+}\right) \cap f\left(U_{2}^{-}\right)=\emptyset$, we can apply Lemma 4.4 and get a regularly equivalent DS-diagram $\Delta_{1}=\left(G_{1}, f_{1}, P_{1} ; e_{1}\right)$. The conditions $f\left(U_{1}^{+}\right)=f\left(V_{1}^{-}\right)$and $f\left(U_{2}^{-}\right)=f\left(V_{2}^{+}\right)$imply that this application of Lemma 4.4 deforms the subword $\Omega(\alpha, \beta)$ into a subword $\Omega_{1}$ of the form

$$
\mathcal{A}\left(\Omega_{1}\right)=V_{1,2 m}^{+} V_{1,2 m-1}^{-} \cdots V_{1,3}^{-} V_{1,2}^{+} V_{1,1}^{-} W_{1}^{+} W_{2}^{-} U_{2}^{-}\left(U_{1}^{+} U_{3}^{+}\right) U_{4}^{-} \cdots U_{2 m-1}^{+} U_{2 m}^{-}
$$

where $V_{1,2 k-1}^{-}$and $V_{1,2 k}^{+}$satisfy that

(i) $V_{1,1}^{-}$differs from $V_{1}^{+}$only by new symbols $x_{1, j}^{-}$produced by the moves,

(ii) $V_{1,2}^{+}$differs from $V_{2}^{+}$only by new symbols $x_{1, j}^{+}$produced by the moves, and

(iii) $V_{1, k}^{ \pm}=V_{k}^{ \pm}$for $k=3,4, \ldots, 2 m$.

Since $\left(f_{1}\left(U_{1}^{+}\right) \cup f_{1}\left(U_{3}^{+}\right)\right) \cap f_{1}\left(U_{4}^{-}\right)=\emptyset$, we can apply Lemma 4.4 again to the subwords $\left(U_{1}^{+} U_{3}^{+}\right)$and $U_{4}^{-}$, and get a regularly equivalent DS-diagram $\Delta_{2}=$ $\left(G_{2}, f_{2}, P_{2} ; e_{2}\right)$ in which the subword $\Omega_{1}$ is deformed into $\Omega_{2}$ such that

$$
\mathcal{A}\left(\Omega_{2}\right)=V_{2,2 m}^{+} V_{2,2 m-1}^{-} \cdots V_{2,3}^{-} V_{2,2}^{+} V_{2,1}^{-} W_{1}^{+} W_{2}^{-} U_{2}^{-} U_{4}^{-}\left(U_{1}^{+} U_{3}^{+} U_{5}^{+}\right) \cdots U_{2 m-1}^{+} U_{2 m}^{-},
$$

which satisfies that

(i) $V_{2,1}^{-}$differs from $V_{1,1}^{+}$only by some of new symbols $x_{2, j}^{-}$produced by the moves,

(ii) $V_{2,2}^{+}=V_{1,2}^{+}$,

(iii) $V_{2,3}^{-}$differs from $V_{1,3}^{-}$only by some of new symbols $x_{2, j}^{-}$produced by the moves,

(iv) $V_{2,4}^{+}$differs from $V_{1,4}^{+}$only by new symbols $x_{2, j}^{+}$produced by the moves, and

(v) $V_{2, k}^{ \pm}=V_{1, k}^{ \pm}$for $k=5,6, \ldots, 2 m$.

Applying Lemma $4.4 m$-times similarly, we get a sequence $\Delta_{k}=\left(G_{k}, f_{k}, P_{k}\right.$; $\left.e_{k}\right)(k=1,2, \ldots, m)$ of regularly equivalent DS-diagrams, in which the subword $\Omega_{0} \equiv \Omega(\alpha, \beta)$ is deformed into a sequence of subwords $\Omega_{1}, \Omega_{2}, \ldots, \Omega_{m}$ such that $\Omega_{k}$ is obtained from $\Omega_{k-1}$ by exchanging the positive subword $\left(U_{1}^{+} U_{3}^{+} \cdots U_{2 k-1}^{+}\right)$ and the negative subword $U_{2 k}^{-}$. By $x_{k, j}^{ \pm}\left(1 \leq j \leq s_{k}\right)$ we denote the new symbols created by the $k$-th application of Lemma 4.4 which deforms $\Omega_{k-1}$ into $\Omega_{k}$, where the number $s_{k}$ is given by

$$
s_{k}=\left(\# U_{2 k}^{-}\right) \times\left(\prod_{j=1}^{k}\left(\# U_{2 j-1}^{+}\right)\right) .
$$


The conditions $f\left(U_{k}^{ \pm}\right)=f\left(V_{k}^{\mp}\right)$ imply that the arrangement of the $m$-th subword $\Omega_{m}$ can be represented as

$$
\begin{aligned}
\mathcal{A}\left(\Omega_{m}\right)= & V_{m, 2 m}^{+} V_{m, 2 m-1}^{-} \cdots V_{m, 3}^{-} V_{m, 2}^{+} V_{m, 1}^{-} W_{1}^{+} \Theta_{1}^{-} \Theta_{2}^{+} \\
& \left(\Theta_{1}^{-}=W_{2}^{-} U_{2}^{-} U_{4}^{-} U_{6}^{-} \cdots U_{2 m}^{-}, \quad \Theta_{2}^{+}=U_{1}^{+} U_{3}^{+} U_{5}^{+} \cdots U_{2 m-1}^{+}\right)
\end{aligned}
$$

and satisfies that

(*i) $V_{m, 2 k-1}^{-}$differs from $V_{2 k-1}^{-}$by some of new symbols $x_{k^{\prime}, j}^{-}$for $k \leq k^{\prime} \leq m$, and (*ii) $V_{m, 2 k}^{+}$differs from $V_{2 k}^{+}$by new symbols $x_{k, j}^{+}$.

Since $f_{m}\left(V_{m, 1}^{-}\right) \cap f_{m}\left(W_{1}^{+}\right)=\emptyset$, we can apply Lemma 4.5 to the DS-diagram $\Delta_{m}$, and get a regularly equivalent DS-diagram $\widetilde{\Delta}_{1}=\left(\widetilde{G}_{1}, \widetilde{f}_{1}, \widetilde{P}_{1} ; \widetilde{e}_{1}\right)$ in which $\Omega_{m}$ is deformed into $\widetilde{\Omega}_{1}$ such that

$$
\mathcal{A}\left(\widetilde{\Omega}_{1}\right)=\widetilde{V}_{1, m}^{+} \widetilde{V}_{1,2 m-1}^{-} \cdots \widetilde{V}_{1,3}^{-}\left(\widetilde{V}_{1,2}^{+} W_{1}^{+}\right)\left(\widetilde{V}_{1,1}^{-} \widetilde{\Theta}_{1,1}^{-}\right) \widetilde{\Theta}_{1,2}^{+} .
$$

The above conditions $\left(*_{\mathrm{i}}\right)$ and $\left({ }^{*} \mathrm{ii}\right)$ imply that $\widetilde{f}_{1}\left(\widetilde{V}_{1,3}^{-}\right) \cap\left(\widetilde{f}_{1}\left(\widetilde{V}_{1,2}^{+}\right) \cup \widetilde{f}_{1}\left(\widetilde{W}_{1}^{+}\right)\right)=\emptyset$. Hence we can apply Lemma 4.5 again, and obtain a regularly equivalent DS-diagram $\widetilde{\Delta}_{2}=\left(\widetilde{G}_{2}, \widetilde{f}_{2}, \widetilde{P}_{2} ; \widetilde{e}_{2}\right)$ in which $\widetilde{\Omega}_{1}$ is deformed into $\widetilde{\Omega}_{2}$ such that

$$
\mathcal{A}\left(\widetilde{\Omega}_{2}\right)=\widetilde{V}_{2, m}^{+} \widetilde{V}_{2,2 m-1}^{-} \cdots \widetilde{V}_{2,5}^{-}\left(\widetilde{V}_{2,4}^{+} \widetilde{V}_{2,2}^{+} W_{1}^{+}\right)\left(\widetilde{V}_{2,3}^{-} \widetilde{V}_{2,1}^{-} \widetilde{\Theta}_{2,1}^{-}\right) \widetilde{\Theta}_{2,2}^{+} .
$$

And the above two conditions show that we can apply Lemma 4.5 to the negative subword $\widetilde{V}_{2,5}^{-}$and the positive subword $\left(\widetilde{V}_{2,4}^{+} \widetilde{V}_{1,2}^{+} W_{1}^{+}\right)$. Similarly the conditions $\left(*_{\mathrm{i}}\right)$ and $\left(*_{\mathrm{ii}}\right)$ enable us to make $m$-times successive applications of Lemma 4.5 , and, after the $m$-th application, we get a regularly equivalent DS-diagram $\widetilde{\Delta}_{m}$ in which the subword $\Omega_{m}$ is deformed into $\widetilde{\Omega}_{m}$ satisfying that

$$
\mathcal{A}\left(\widetilde{\Omega}_{m}\right)=\left(\widetilde{V}_{m, 2 m}^{+} \widetilde{V}_{m, 2 m-2}^{+} \cdots \widetilde{V}_{m, 2}^{+} W_{1}^{+}\right)\left(\widetilde{V}_{m, 2 m-1}^{-} \widetilde{V}_{m, 2 m-3}^{-} \cdots \widetilde{V}_{m, 1}^{-} \widetilde{\Theta}_{m, 1}^{-}\right) \widetilde{\Theta}_{m, 2}^{+}
$$

Consequently the original DS-diagram $\Delta=(G, f, P ; e)$ with an E-data $\mathcal{E}(\Delta)=$ $\Omega(\alpha, \beta) \Phi$ can be deformed by regular moves into a DS-diagram $\Delta^{\prime}=\left(G^{\prime}, f^{\prime}, P^{\prime} ; e^{\prime}\right)$ $\left(\Delta^{\prime} \equiv \widetilde{\Delta}_{m}\right)$ having an arrangement of the form

$$
\left.\mathcal{A}\left(\Delta^{\prime}\right)=X_{1}^{+} X_{2}^{-} X_{3}^{+} \Phi \quad \text { (the arrangement for } \Phi \text { is also denoted by } \Phi\right),
$$

and the negative subword $X_{2}^{-}$and the positive subwords $X_{1}^{+}, X_{3}^{+}$satisfy $f^{\prime}\left(X_{2}^{-}\right)=$ $f^{\prime}\left(X_{1}^{+}\right) \cup f^{\prime}\left(X_{3}^{+}\right)$. Different applications of Lemmas 4.4 and 4.5 lead us to another regularly equivalent DS-diagram $\Delta^{\prime \prime}=\left(G^{\prime \prime}, f^{\prime \prime}, P^{\prime \prime} ; e^{\prime \prime}\right)$ whose E-data has an arrangement of the form

$$
\mathcal{A}\left(\Delta^{\prime \prime}\right)=Y_{1}^{-} Y_{2}^{+} Y_{3}^{-} \Phi, \quad f^{\prime \prime}\left(Y_{2}^{+}\right)=f^{\prime \prime}\left(Y_{1}^{-}\right) \cup f^{\prime \prime}\left(Y_{3}^{-}\right) .
$$


Indeed, $m$-times applications of Lemma 4.5 on $\left(V_{2 m}^{+} V_{2 m-1}^{-} \cdots V_{1}^{-}\right)$and subsequent $m$-times applications of Lemma 4.4 yield a required DS-diagram $\Delta^{\prime \prime}$. Hence we have the following lemma.

Lemma 4.7. Let $\Delta=(G, f, P ; e)$ a DS-diagram having an E-data of the form $\mathcal{E}(\Delta)=\Omega(\alpha, \beta) \Phi$. Then there exist two DS-diagrams $\Delta^{\prime}=\left(G^{\prime}, f^{\prime}, P^{\prime} ; e^{\prime}\right)$ and $\Delta^{\prime \prime}=\left(G^{\prime \prime}, f^{\prime \prime}, P^{\prime \prime} ; e^{\prime \prime}\right)$ such that both $\Delta^{\prime}$ and $\Delta^{\prime \prime}$ are regularly equivalent to $\Delta$, and their E-data have arrangements of the form

$$
\begin{array}{ll}
\mathcal{A}\left(\Delta^{\prime}\right)=X_{1}^{+} X_{2}^{-} X_{3}^{+} \Phi, & f^{\prime}\left(X_{2}^{-}\right)=f^{\prime}\left(X_{1}^{+}\right) \cup f^{\prime}\left(X_{3}^{+}\right) \quad \text { and } \\
\mathcal{A}\left(\Delta^{\prime \prime}\right)=Y_{1}^{-} Y_{2}^{+} Y_{3}^{-} \Phi, & f^{\prime \prime}\left(Y_{2}^{+}\right)=f^{\prime \prime}\left(Y_{1}^{-}\right) \cup f^{\prime \prime}\left(Y_{3}^{-}\right) .
\end{array}
$$

4.4. Proof of Lemma 3.2. According to Theorem 4.2, it is sufficient for the proof of Lemma 3.2 to show that a DS-diagram $\Delta=(G, f, P ; e)$ having an E-data $\mathcal{E}(\Delta)=\Omega\left(\alpha_{1}, \beta_{1} ; x\right) \Omega\left(\alpha_{2}, \beta_{2} ; y\right)$ can be deformed by regular moves into a DS-diagram of block number 1 .

Applying Lemma 4.7 twice, we obtain a DS-diagram $\Delta^{\prime}=\left(G^{\prime}, f^{\prime}, P^{\prime} ; e^{\prime}\right)$ which is regularly equivalent to $\Delta$, and has an arrangement

$$
\mathcal{A}\left(\Delta^{\prime}\right)=\left(X_{1}^{+} X_{2}^{-} X_{3}^{+}\right)\left(Y_{1}^{-} Y_{2}^{+} Y_{3}^{-}\right) .
$$

Since $f^{\prime}\left(X_{3}^{+}\right) \cap f^{\prime}\left(Y_{1}^{-}\right)=\emptyset$, we can apply Lemma 4.4, and obtain a DS-diagram $\Delta^{\prime \prime}=\left(G^{\prime \prime}, f^{\prime \prime}, P^{\prime \prime} ; e^{\prime \prime}\right)$ having an arrangement

$$
\mathcal{A}\left(\Delta^{\prime \prime}\right)=X_{1}^{+}\left(\tilde{X}_{2}^{-} Y_{1}^{-}\right)\left(X_{3}^{+} \tilde{Y}_{2}^{+}\right) Y_{3}^{-}
$$

which satisfies $f^{\prime \prime}\left(X_{1}^{+}\right) \cap f^{\prime \prime}\left(Y_{3}^{-}\right)=\emptyset$. Hence, applying Lemma 4.6 to the positive block $X_{1}^{+}$and the negative block $Y_{3}^{-}$which are mutually adjacent in a cyclical order, we obtain a regularly equivalent DS-diagram whose block number is 1 . This completes the proof of Lemma 3.2.

4.5. Proof of Theorem 4.1. It is well known that the Heegaard genus of a Seifert fibered space $M=\Sigma\left(3 ;\left(\alpha_{k}, \beta_{k}\right)\right)$ is two. Furthermore we have shown $H G(M) \leq B l(M)$ in Theorem 2.6. Hence, in order to prove Theorem 4.1, it is enough to show that we can deform the DS-diagram $\Delta$ given by the E-data (4.2) into a DS-diagram $\Delta^{\prime}$ such that $\Delta \cong \Delta^{\prime}$ and $b l\left(\Delta^{\prime}\right)=2$.

Let $\Delta=(G, f, P ; e)$ be the DS-diagram whose E-data $\mathcal{E}(\Delta)$ is given by the formula (4.2). Applying Lemma 4.7 three times, we get a regularly equivalent DS-diagram $\Delta_{0}=\left(G_{0}, f_{0}, P_{0} ; e_{0}\right)$ having an arrangement of E-data

$$
\mathcal{A}\left(\Delta_{0}\right)=\left(a_{1}^{-} a_{3}^{+}\right) X_{1}^{+} X_{2}^{-} X_{3}^{+}\left(a_{2}^{-} a_{1}^{+}\right) Y_{1}^{+} Y_{2}^{-} Y_{3}^{+}\left(a_{3}^{-} a_{2}^{+}\right) Z_{1}^{-} Z_{2}^{+} Z_{3}^{-},
$$

where the subwords in this representation satisfy that $f_{0}\left(X_{1}^{+}\right) \cup f_{0}\left(X_{3}^{+}\right)=f_{0}\left(X_{2}^{-}\right)$, $f_{0}\left(Y_{1}^{+}\right) \cup f_{0}\left(Y_{3}^{+}\right)=f_{0}\left(Y_{2}^{-}\right)$and $f_{0}\left(Z_{1}^{-}\right) \cup f_{0}\left(Z_{3}^{-}\right)=f_{0}\left(Z_{2}^{+}\right)$. In what follows, we 
make subsequent applications (I)-(IV) of Lemmas 4.4 and 4.5, and we will finally get a DS-diagram $\Delta^{\prime}$ with $b l\left(\Delta^{\prime}\right)=2$. In each step of applications of the lemmas, we must decide the positions where the new symbols appear, and we must carefully check the condition for the lemma to be applicable.

(I) Application of Lemma 4.4 to subwords $X_{3}^{+}$and $a_{2}^{-}$.

First apply Lemma 4.4 to the positive subword $X_{3}^{+}$and the negative subword $a_{2}^{-}$of $\mathcal{A}\left(\Delta_{0}\right)$. Then we get a regularly equivalent DS-diagram $\Delta_{1}=\left(G_{1}, f_{1}, P_{1} ; e_{1}\right)$ having an arrangement

$$
\mathcal{A}\left(\Delta_{1}\right)=\left(a_{1}^{-} a_{3}^{+}\right) X_{1}^{+} \tilde{X}_{2}^{-} a_{2}^{-} X_{3}^{+} a_{1}^{+} Y_{1}^{+} Y_{2}^{-} Y_{3}^{+} a_{3}^{-} \widetilde{A}_{2}^{+} Z_{1}^{-} Z_{2}^{+} Z_{3}^{-} .
$$

The subwords $X_{2}^{-}$and $a_{2}^{+}$of $\mathcal{A}\left(\Delta_{0}\right)$ were deformed into $\widetilde{X}_{2}^{-}$and $\widetilde{A}_{2}^{+}$respectively, which include new symbols caused by an application of Lemma 4.4 .

(II) Application of Lemma 4.4 to subwords $Y_{3}^{+}$and $a_{3}^{-}$.

Make similar application of Lemma 4.4 to the positive subword $Y_{3}^{+}$and the negative subword $a_{3}^{-}$of $\mathcal{A}\left(\Delta_{1}\right)$. Then we obtain a regularly equivalent DS-diagram $\Delta_{2}=\left(G_{2}, f_{1}, P_{2} ; e_{2}\right)$ which has an arrangement

$$
\mathcal{A}\left(\Delta_{2}\right)=a_{1}^{-} \widetilde{A}_{3}^{+} X_{1}^{+} \widetilde{X}_{2}^{-} a_{2}^{-} X_{3}^{+} a_{1}^{+} Y_{1}^{+} \widetilde{Y}_{2}^{-} a_{3}^{-}\left(Y_{3}^{+} \widetilde{A}_{2}^{+}\right) Z_{1}^{-} Z_{2}^{+} Z_{3}^{-} .
$$

This operation deforms the subwords $Y_{2}^{-}$and $a_{3}^{+}$of $\mathcal{A}\left(\Delta_{1}\right)$ into $\widetilde{Y}_{2}^{-}$and $\widetilde{A}_{3}^{+}$respectively, which include new symbols produced by an application of the lemma.

(III) Application of Lemma 4.4 to subwords $\left(Y_{3}^{+} \widetilde{A}_{2}^{+}\right)$and $Z_{1}^{-}$.

Next apply Lemma 4.4 to the positive subword $\left(Y_{3}^{+} \widetilde{A}_{2}^{+}\right)$and the negative subword $Z_{1}^{-}$of $\mathcal{A}\left(\Delta_{2}\right)$. Then we have a regularly equivalent DS-diagram $\Delta_{3}=$ $\left(G_{3}, f_{3}, P_{3} ; e_{3}\right)$ having an arrangement

$$
\mathcal{A}\left(\Delta_{3}\right)=a_{1}^{-} \widetilde{A}_{3}^{+} X_{1}^{+} \widehat{X}_{2}^{-} \widetilde{B}_{2}^{-} X_{3}^{+} a_{1}^{+} Y_{1}^{+} \widehat{Y}_{2}^{-} a_{3}^{-} Z_{1}^{-} Y_{3}^{+} \widetilde{A}_{2}^{+} \widetilde{Z}_{2}^{+} Z_{3}^{-} .
$$

This operation deforms the four subwords $a_{2}^{-}, \widetilde{X}_{2}^{-}, \widetilde{Y}_{2}^{-}$, and $Z_{2}^{+}$of $\mathcal{A}\left(\Delta_{2}\right)$ into $\widetilde{B}_{2}^{-}$, $\widehat{X}_{2}^{-}, \widehat{Y}_{2}^{-}$and $\widetilde{Z}_{2}^{+}$respectively, which include new symbols caused by this operation. By a cyclical permutation, we have a blockwise representation

$$
\mathcal{A}\left(\Delta_{3}\right)=\left(Z_{3}^{-} a_{1}^{-}\right)\left(\widetilde{A}_{3}^{+} X_{1}^{+}\right)\left(\widehat{X}_{2}^{-} \widetilde{B}_{2}^{-}\right)\left(X_{3}^{+} a_{1}^{+} Y_{1}^{+}\right)\left(\widehat{Y}_{2}^{-} a_{3}^{-} Z_{1}^{-}\right)\left(Y_{3}^{+} \widetilde{A}_{2}^{+} \widetilde{Z}_{2}^{+}\right),
$$

which has three positive blocks and three negative blocks.

(IV) Application of Lemma 4.5 to subwords $\left(Z_{3}^{-} a_{1}^{-}\right)$and $\left(\widetilde{A}_{3}^{+} X_{1}^{+}\right)$.

Finally apply Lemma 4.5 (or Lemma 4.6$)$ to the negative block $\left(Z_{3}^{-} a_{1}^{-}\right)$and the positive block $\left(\widetilde{A}_{3}^{+} X_{1}^{+}\right)$of $\mathcal{A}\left(\Delta_{3}\right)$. Since $f_{3}\left(Z_{3}^{-} a_{1}^{-}\right) \cap f_{3}\left(\widetilde{A}_{3}^{+} X_{1}^{+}\right)=\emptyset$, we can really make this application. As a consequence of the application of the lemma, we get a regularly equivalent DS-diagram $\Delta_{4}$ satisfying $b l\left(\Delta_{4}\right)=2$. 
This completes the proof of Theorem 4.1.

\section{Problems on block numbers}

We have shown that $B l(M)=H G(M)$ for $M=L(p, q)(p>1)$ and for $M=\Sigma\left(3 ;\left(\alpha_{k}, \beta_{k}\right)\right)$. There naturally arises the following problem.

Problem 1. Is $B l(M)=H G(M)$ for any orientable closed 3-manifold $M$ except for $S^{2} \times S^{1}$ and $S^{3}$ ?

In the forthcoming paper, we will give an affirmative answer to this problem in the case of $H G(M)=2$. However Problem 1 is quite open for the case of $H G(M) \geq 3$.

It was shown in this paper that $B l^{*}(\mathfrak{C})=B l(M)$ for canonical oriented combed 3-manifolds $\mathfrak{C}$ on some Seifert fibered spaces $M$. On the other hand, a systematic method for describing E-data of canonical oriented combed 3-manifolds on all Seifert fibered spaces is established in [13]. This method together with our proof of Theorem 4.1 strongly implies an affirmative answer to the following problem.

Problem 2. Is $B l^{*}(\mathfrak{C})=B l(M)$ for any canonical oriented combed 3manifold $\mathfrak{C}$ on a Seifert fibered space $M$ ?

Moreover we have shown that, for the case of $M=L(p, q)$ and $\mathfrak{C}=(M, X)$, $B l^{*}(\mathfrak{C})=B l(M)$ only if $\mathfrak{C}$ is canonical. Generally we can ask that

Problem 3. Is $B l^{*}(\mathfrak{C})>B l(M)$ for any non-canonical oriented combed manifold $\mathfrak{C}$ on a Seifert fibered space $M$ ? How about $M=\Sigma\left(3 ;\left(\alpha_{k}, \beta_{k}\right)\right)$ ?

What is a "canonical" vector field for a non-Seifert manifold? From a viewpoint of the block number, this question is formulated as the following problem.

Problem 4. Which non-singular vector field $X$ on $M$ satisfy $B l^{*}(\mathfrak{C})=$ $B l(M)$ for the oriented combed 3-manifold $\mathfrak{C}=(M, X)$ ? How about a graph manifold $M$, and how about a hyperbolic 3-manifold $M$ ?

It is said that the so called "Haken's theorem of finiteness" gives an algorithm to decide the Heegaard genus. Analogously we can propose the following problem.

Problem 5. Is there any algorithm to decide the block number $B l^{*}(\mathfrak{C}(\Delta))$ for a given DS-diagram $\Delta$ with E-cycle?

In our proof of Theorem 4.1, the only essential tool for reducing the block number is Lemma 4.6. Hence, as the most optimistic version of Problem 5, we can consider the following problem.

Problem 6. Is $B l^{*}(\mathfrak{C}(\Delta))=b l(\Delta)$ if $\Delta$ admits no application of Lemma 4.6? 
It is maybe too optimistic to expect an affirmative answer to Problem 6 . However a counterexample may give an interesting example of an oriented combed 3-manifold.

\title{
References
}

[1] R. Benedetti and C. Petronio, Branched Standard Spines of 3-Manifolds, Springer LNM 1653, (1997).

[2] H. Ikeda, Acyclic fake surfaces, Topology, 10 (1971), 9-36.

[3] H. Ikeda, Acyclic fake surfaces which are spines of 3-manifolds, Osaka J. Math., 9 (1972), 391-408.

[4] H. Ikeda, Identification maps on $S^{2}$, Kobe J. Math., 2 (1985), 163-167.

[ 5 ] H. Ikeda and Y. Inoue, Invitation to DS-diagrams, Kobe J. Math., 2 (1985), 169-186.

[6] H. Ikeda, DS-diagrams with E-cycle, Kobe J. Math., 3 (1986), 103-112.

[ 7 ] H. Ikeda and M. Kouno, Like a flow-spine, Kobe J. Math., 8 (1991), 93-100.

[8] I. Ishii, Flows and spines, Tokyo J. Math., 9 (1986), 505-525.

[9] I. Ishii, Combinatorial construction of a non-singular flow on a 3-manifold, Kobe J. Math., 3 (1987), 201-208.

[10] I. Ishii, Flow-spines and Seifert fibred structure of 3-manifolds, Tokyo J. Math., 11 (1988), 95-104.

[11] I. Ishii, Moves for flow-spines and topological invariants of 3-manifolds, Tokyo J. Math., 15 (1992), 297-312.

[12] I. Ishii, Fake braid group and 3-manifolds, Geometry and Its Applications (ed. by T. Nagano et al.), World Scientific (1993), 51-58.

[13] T. Taniguchi, K. Tsuboi and M. Yamashita, Systematic singular triangulations for all Seifert manifolds, to appear in Tokyo J. Math.

\author{
MARIKO ENDOH \\ Department of MATHEMATics \\ Faculty of Science and Technology \\ SOPHIA UNIVERSITY \\ Kiol-Cho, Chiyoda-ku, Tokyo 102-8554 \\ JAPAN \\ E-mail: mariko@mm.sophia.ac.jp \\ IPPEI ISHII \\ Department of Mathematics \\ Faculty of Science and Technology \\ KeIO UNIVERSITY \\ HгYoshi, КонокU-KU, Үоконама 223-8522 \\ JAPAN \\ E-mail: ishii@math.keio.ac.jp
}

